ESAIM: M2AN 47 (2013) 903-932

DOI: $10.1051 / \mathrm{m} 2 \mathrm{an} / 2012061$
ESAIM: Mathematical Modelling and Numerical Analysis

www.esaim-m2an.org

\title{
STABILITY ANALYSIS OF THE INTERIOR PENALTY DISCONTINUOUS GALERKIN METHOD FOR THE WAVE EQUATION
}

\author{
Cyril Agut ${ }^{1}$ And Julien Diaz ${ }^{2}$
}

\begin{abstract}
We consider here the Interior Penalty Discontinuous Galerkin (IPDG) discretization of the wave equation. We show how to derive the optimal penalization parameter involved in this method in the case of regular meshes. Moreover, we provide necessary stability conditions of the global scheme when IPDG is coupled with the classical Leap-Frog scheme for the time discretization. Numerical experiments illustrate the fact that these conditions are also sufficient.
\end{abstract}

Mathematics Subject Classification. 35L05, 65M12, 65M60.

Received April 14, 2011. Revised June 2, 2012.

Published online April 17, 2013.

\section{INTRODUCTION}

The accurate numerical solution to acoustic, elastodynamic or electromagnetic wave equations is required in a number of important applications such as medical imaging, seismic imaging, radar or earthquakes simulations. In many cases, the equations have to be solved in very large domains with strong heterogeneities. Therefore, one has to use sophisticated discretization methods to compute the most accurate solution at the smallest computational cost.

Finite Differences Methods, which are widely used because of their small computational cost and the simplicity of their implementation, are not adapted to deal with strong heterogeneities. Indeed, they rely on structured grids, which can not accurately approximate the shape of the various layers of the domain. Finite Elements Methods (FEM) are more adapted to this kind of problems since they allow for the use of unstructured grids. Among all existing FEM, the Spectral Element Method [7,9,17,19], is probably one of the most efficient methods to solve the wave equation since the resulting mass matrix is diagonal. Hence, it can be easily coupled to explicit time-schemes, which involves the inversion of the mass matrix at each time step. However, SEM requires the use of quadrilateral (in 2D) or hexahedral (in 3D) meshes which can be difficult to generate for realistic applications. Let us however mention that SEM can be extended to handle triangular meshes [8], but this requires additional degrees of freedom and the implementation is more complex. Moreover, as far as we know, the extension to tetrahedral meshes has not been proposed yet.

\footnotetext{
Keywords and phrases. Discontinuous Galerkin, penalization coefficient, CFL condition, wave equation.

1 LMAP, University of Pau, INRIA Project-Team Magique-3D, France. cyril.agut@orange.fr

2 INRIA Project-Team Magique-3D, LMAP, University of Pau, France
} 
Discontinuous Galerkin Methods are more and more popular for solving the wave equation since they lead to block-diagonal mass matrices without the help of quadrature formula. Moreover, they can be used with any type of meshes and even allow for the variation of the physical parameters inside the cells of the mesh (provided that the variation of each physical quantity can be approximated by polynomial functions). DGM are also naturally adapted to parallel computing since all volume integrals are computed locally and the communications between the cells are ensured by integrals over the faces of the elements. In [4], Arnold et al. provide a detailed review of the various Discontinuous Galerkin approximations of the Laplacian operator. They show that the so-called Interior Penalty Discontinuous Galerkin Method (IPDGM), also known as Symmetric Interior Penalty (SIP) [3], is one of the most suitable since it is stable and adjoint consistent, which guarantees the optimal order of convergence of the scheme. This explains why this method has been succesfully used to solve Helmholtz equation $[2,6]$ and the wave equation $[2,15,16]$. Comparisons of the performances of IPDGM and SEM can be found in $[5,11]$. It is worth noting that the first paper concluded that the performances of SEM are better than IDPGM when IPDGM is applied to structured grids composed of squares, while the second paper shows that the performances of IPDGM are better when it is applied to triangular meshes. However, the methodologies were slightly different. In the first paper, the computational costs and the accuracy of both methods are compared for a given mesh. It appeared that the computational costs of SEM (computational time and storage) were smaller than IPDG but that the accuracy of IPDG was better. In the second paper the computational cost are compared for a given accuracy and it appeared that the computational costs of IPDG were smaller for high order element (polynomial degree greater than two).

Nevertheless, in spite of all its interesting properties, IPDGM still suffers from two difficulties. The first one is the determination of the penalization parameter, which penalizes the discontinuities of the solution through the faces. The accurate determination of the optimal parameter is crucial, since a too small value leads to instabilities while a too large value could (strongly) hamper the CFL (Courant-Friedrichs-Lewy) condition, which gives the maximal time step that can be used to ensure the stability of the scheme. In [2], Ainsworth, Monk and Muniz conjectured a minimal value of the penalization parameters, depending on $p$, the polynomial degree of the basis functions and on the size of the elements. They proved their conjecture up to $p=3$. The extension of this result to $p>3$ and to unstructured meshes, is still to be done. The second difficulty is the determination of the CFL condition. It is well-known that this condition decreases when the penalization parameter increases, but no analytical formula has been proposed yet. The aim of this paper is (a) to prove the conjecture of Ainsworth, Monk and Muniz up to $p=5$; (b) to propose a solution methodology to prove it for a given $p$; and (c) to provide an analytic formula linking the CFL condition to the penalization parameter. We restrict ourselves to the cases of structured meshes composed of segments (in 1D), squares (in 2D) or cubes (in 3D). In Section 1, we recall the IPDG discretization of the wave equation. In Section 2, we propose two theorems, the first one provides explicit necessary stability conditions on the penalization parameter and the time step while the second one provides a more restrictive but implicit necessary stability condition. The proof of these theorems in the one dimensional case is given in Section 3. Section 4 is devoted to the proof in the three dimensional case and contains a discussion on the adaptation of the theorems to structured meshes composed of rectangles or parallelepipeds. We do not present the proof in the two dimensional case, but it can be adapted without any difficulties from the three dimensional case. Finally, we present numerical results in Section 5 that illustrate the fact that the stability condition is actually necessary and sufficient for numerical applications.

\section{INTERIOR PENALTY DISCONTINUOUS GALERKIN DISCRETIZATION OF THE WAVE EQUATION}

In this section, we recall the so called Interior Penalty Discontinuous Galerkin method applied to the acoustic wave equation in homogeneous bounded media $\Omega \subset \mathbb{R}^{d}, d=1,2,3$. For the sake of simplicity, we impose homogeneous Dirichlet boundary conditions on the boundary $\Gamma:=\partial \Omega$ but this study can be extended to 
Neumann boundary conditions without major difficulties.

$$
\begin{cases}\text { Find } u: \Omega \times[0, T] \mapsto \mathbb{R} \text { such that : } & \\ \frac{1}{\mu} \frac{\partial^{2} u}{\partial t^{2}}-\operatorname{div}\left(\frac{1}{\rho} \nabla u\right)=f & \text { in } \quad \Omega \times] 0, T] \\ u(x, 0)=u_{0}, \quad \frac{\partial u}{\partial t}(x, 0)=u_{1} & \text { in } \quad \Omega, \\ u=0 & \text { on } \quad \partial \Omega .\end{cases}
$$

where $u$ stands for the displacement, $\mu$ is the compressibility modulus, $\rho$ is the density and $f$ is the source term. In this paper, $\rho$ and $\mu$ are assumed to be constant, but in practical applications they are usually assumed to be piecewise constant or piecewise polynomial.

We introduce a triangulation $\mathcal{T}_{h}$ of $\Omega$ and the following space of approximation with piecewise discontinuous polynomial functions :

$$
V_{h}:=\left\{v \in L^{2}(\Omega): v_{\mid K} \in P^{p}(K), \forall K \in \mathcal{T}_{h}\right\} .
$$

The IPDG method can be applied with piecewise constant finite elements $(p=0)$ as it is mentionned in [2] but in practice we consider polynomial degrees greater than or equal to one.

The set of the mesh faces is denoted $\mathcal{F}_{h}$ which is partitionned into two subsets $\mathcal{F}_{h}^{i}$ and $\mathcal{F}_{h}^{b}$ corresponding respectively to the interior faces and those located on the boundary. For $F \in \mathcal{F}_{h}^{i}$, we denote by $K^{+}$and $K^{-}$the two elements sharing $F$ and we define $\nu$ as the unit outward normal vector pointing from $K^{+}$to $K^{-}$. Moreover, $v^{ \pm}$represents the restriction of a function $v$ to the element $K^{ \pm}$and we define the jump and the average of a piecewise smooth function $v \in V_{h}$ over $F \in \mathcal{F}_{h}^{i}$ as

$$
\llbracket v \rrbracket=v^{+}-v^{-}, \quad\{\{v\}\}=\frac{v^{+}+v^{-}}{2} .
$$

For $F \in \mathcal{F}_{h}^{b}$, we define $\llbracket v \rrbracket=v$ and $\{\{v\}\}=v$.

The IPDG discretization of (2.1) reads as

$$
\left\{\begin{array}{l}
\text { Find } u_{h} \in V_{h} \text { such that, } \forall v_{h} \in V_{h}: \\
\sum_{K \in \mathcal{T}_{h}} \int_{K} \frac{1}{\mu} \partial_{t}^{2} u_{h} v_{h} \mathrm{~d} x=-a_{h}\left(u_{h}, v_{h}\right)+\sum_{K \in \mathcal{T}_{h}} \int_{K} f v_{h} \mathrm{~d} x .
\end{array}\right.
$$

where $a_{h}$ is a bilinear form defined by

$$
a_{h}\left(u_{h}, v_{h}\right)=B_{\mathcal{T}_{h}}\left(u_{h}, v_{h}\right)-\mathcal{I}\left(u_{h}, v_{h}\right)-\mathcal{I}\left(v_{h}, u_{h}\right)+B_{S}\left(u_{h}, v_{h}\right),
$$

with

$$
\begin{gathered}
B_{\mathcal{T}_{h}}\left(u_{h}, v\right)=\sum_{K \in \mathcal{T}_{h}} \int_{K} \frac{1}{\rho} \nabla u_{h} \nabla v, \quad \mathcal{I}\left(u_{h}, v\right)=\sum_{F \in \mathcal{F}_{h}^{i}} \int_{F} \llbracket v \rrbracket\left\{\left\{\frac{1}{\rho} \nabla u_{h} \cdot \nu\right\}\right\}, \\
B_{S}\left(u_{h}, v\right)=\sum_{F \in \mathcal{F}_{h}^{i}} \int_{F} \gamma \llbracket u_{h} \rrbracket \llbracket v \rrbracket .
\end{gathered}
$$

The bilinear form $B_{S}$ is devoted to enforce the coercivity of $a_{h}$ and the penalization function $\gamma$ is defined on each interior face $F$ by

$$
\gamma=\frac{\alpha}{\xi_{F}}
$$


where $\alpha$ is a positive parameter. There are many definitions of the function $\xi_{F}$ in the litterature. The most commonly used are:

- $\xi_{F}=h(F)$ where $h(F)$ denotes the diameter of $F$. See for instance $[2,4,12,16]$. It is worth noting that this definition does not make sense in 1D.

- $\xi_{F}=\min \left(h\left(K^{+}\right), h\left(K^{-}\right)\right)$where $h\left(K^{ \pm}\right)$is the diameter of $K^{ \pm}$. See for instance [15].

- $\xi_{F}=\min \left(\rho\left(K^{+}\right), \rho\left(K^{-}\right)\right)$where $\rho\left(K^{ \pm}\right)$is the diameter of the inscribed circle (or sphere) of $K^{ \pm}$. See for instance $[20]$.

Whatever the definition of $\xi_{F}$, there exists $\alpha_{0}^{p}>0$ such that the coercivity of $a_{h}$ is ensured for all $\alpha \geq \alpha_{0}^{p}$. Obviously, the optimal parameter $\alpha_{0}$ depends on the choice of the basis functions of $V_{h}$, but also on $\xi_{F}$. It has been shown by Shabazi in [20], using inverse inequalities proposed in [22], that the third definition was the most appropriate for triangular meshes. In [2], it has been shown that, on meshes of squares or cubes, using the first definition of $\xi_{F}$, the optimal parameter is $\alpha_{0}^{p}=p(p+1) / 2$ for $p=0, \ldots, 3$ and they conjectured this relation for $p \geq 4$. It is worth noting that, for such meshes, the first and the third definition of $\xi_{F}$ are equivalent. More recently, Epshteyn and Rivière [12] have proposed a more sophisticated definition of $\xi_{F}$ for meshes of triangles and tetrahedra. This definition does not only involve the length of the edge or the surface of the face, but also the minimal angle of elements $K^{+}$and $K^{-}$.

At this point, we choose not to make explicit the expression for $\xi_{F}$. This will be done in the next section.

We refer to $[2,4,15]$ for more details on the properties of the bilinear form $a_{h}$.

Considering $\left\{\varphi_{i}\right\}_{i=1, \ldots, m}$ the classical discontinuous Lagrange basis functions of degree $p$ of $V_{h}$, where $m$ denotes the number of degrees of freedom of the problem, we obtain the following linear system:

$$
\partial_{t}^{2} U=M^{-1} K U+M^{-1} F
$$

where

$$
(M)_{i, j}=\sum_{K \in \mathcal{T}_{h}} \int_{K} \varphi_{i} \varphi_{j},(K)_{i, j}=a_{h}\left(\varphi_{i}, \varphi_{j}\right),(F)_{i}=\sum_{K \in \mathcal{T}_{h}} \int_{K} f \varphi_{i} .
$$

Now, we have to discretize in time. Using the well known Leap-Frog scheme, we obtain the following fully discretized scheme:

$$
\frac{U^{n+1}-2 U^{n}+U^{n-1}}{\Delta t^{2}}=-M^{-1} K U^{n}+M^{-1} F^{n} .
$$

Since (2.5) is an explicit scheme, its $L^{2}$ stability is constrained by a CFL condition. It is well known [16] that this CFL condition decreases when $\alpha$ increases and behaves as $1 / \sqrt{\alpha}$ for large $\alpha$. However, no explicit formula of the CFL condition has been proposed yet. This is the objective of the next section.

Let us note that, even if all the results are related to the second-order Leap-Frog scheme, they can be easily extended to higher-order schemes obtained by the modified equation technique [10,21], also known as Lax-Wendroff schemes [18]. Indeed, the CFL condition of this schemes is simply the CFL condition of the Leap-Frog scheme multiplied by a factor depending on the order of the scheme (see [14] for more details).

\section{Stability ANALYSis}

In this section, we first prove necessary conditions on $\gamma$ and $\Delta t$ ensuring the $L^{2}$-stability of scheme $(2.5)$. This theorem provides an explicit dependence of $\Delta t$ with respect to $\gamma$ and $h$. Next we propose a more restrictive necessary stability condition. In this second theorem, the dependence of $\Delta t$ with respect to $\gamma$ is no longer explicit. However the condition can be numerically computed using the roots of a polynomial of degree $2 p$. We assume here that the domain $\Omega$ is unbounded $\left(\Omega=\mathbb{R}^{d}\right)$ and uniformly meshed by segments (if $d=1$ ), squares (if $d=2$ ) or cubes (if $d=3$ ). We denote by $h$ the length of segments in $1 \mathrm{D}$ and the length of edges of squares and cubes in 2D and 3D. We define $c$ the wave velocity by $c=\sqrt{\frac{\mu}{\rho}}$.

First of all, let us recap the definition of the $L^{2}$-stability. 
Definition 3.1. A scheme is $L^{2}$-stable if and only if its solution satisfies $\left\|U^{n}\right\|_{L^{2}} \leq C n \Delta t$.

The necessary stability conditions are given by the following theorem.

Theorem 3.2. Let $1 \leq p \leq 5$ and let us set $\alpha_{p}^{0}=\frac{p(p+1)}{2}$. For the scheme (2.5) to be $L^{2}$-stable, the two following conditions have to be satisfied,

$$
\gamma \geq \frac{\alpha_{p}^{0}}{h}
$$

and, writing $\gamma=\alpha / h$,

$$
\sqrt{d} \frac{c \Delta t}{h} \leq\left\{\begin{aligned}
C_{1, p} & \text { if } \alpha \leq \alpha_{1, p} \\
C_{2, p}(\alpha) & \text { if } \alpha>\alpha_{1, p}
\end{aligned}\right.
$$

where $\alpha_{1, p}, C_{1, p}$ and $C_{2, p}(\alpha)$ are defined with respect to the polynomial degree $p$ as:

\begin{tabular}{|l|c|c|c|}
\hline$p$ & $\alpha_{1, p}$ & $C_{1, p}$ & $C_{2, p}(\alpha)$ \\
\hline 1 & 2 & $\frac{\sqrt{3}}{3} \simeq 0.577$ & $\frac{1}{\sqrt{3(\alpha-1)}}$ \\
\hline 2 & $\frac{27}{5}=5.4$ & $\frac{1}{\sqrt{15}} \simeq 0.258$ & $\sqrt{\frac{2}{-15+6 \alpha+\left(405-240 \alpha+36 \alpha^{2}\right)^{1 / 2}}}$ \\
\hline 3 & $\frac{2 \sqrt{1605}+393}{49} \simeq 9.65$ & $\sqrt{\frac{2}{45+\sqrt{1605}}} \simeq 0.153$ & $\sqrt{\frac{2}{-45+10 \alpha+\left(4545-1320 \alpha+100 \alpha^{2}\right)^{1 / 2}}}$ \\
\hline 4 & $\alpha_{1,4} \simeq 14.7$ & $\sqrt{\frac{2}{3(35+\sqrt{805})}} \simeq 0.103$ & $\sqrt{\frac{1}{2 \sqrt{5} g_{4,1}(\alpha) g_{4,2}(\alpha)+5 \alpha-35}}$ \\
\hline 5 & $\alpha_{1,5} \simeq 20.8$ & $\sqrt{\frac{1}{10 \sqrt{133} \cos \left(g_{5}\right)+70}} \simeq 0.074$ & $\sqrt{\frac{1}{2 \sqrt{7} g_{5,1}(\alpha) g_{5,2}(\alpha)+7(\alpha-10)}}$ \\
\hline
\end{tabular}

where, for the case $p=4$, we have

$$
\left\{\begin{array}{l}
g_{4,1}(\alpha)=\left(518-98 \alpha+5 \alpha^{2}\right)^{\frac{1}{2}}, \\
g_{4,2}(\alpha)=\cos \left(\frac{1}{3} \arccos \left(\frac{1}{10} g_{4,3}(\alpha) \frac{\sqrt{5}}{g_{4,1}^{3}(\alpha)}\right)\right), \\
g_{4,3}(\alpha)=-47705+14574 \alpha-1470 \alpha^{2}+50 \alpha^{3}
\end{array}\right.
$$

and for the polynomials of degree 5 ,

$$
\left\{\begin{aligned}
g_{5} & =\frac{1}{3} \arccos \left(\frac{10447}{126350} \sqrt{133}\right) \\
g_{5,1}(\alpha) & =\left(1555-200 \alpha+7 \alpha^{2}\right)^{\frac{1}{2}}, \\
g_{5,2}(\alpha) & =\cos \left(\frac{1}{3} \arccos \left(\frac{1}{14} g_{5,3}(\alpha) \frac{\sqrt{7}}{g_{5,1}^{3}(\alpha)}\right)\right), \\
g_{5,3}(\alpha) & =-299825+61440 \alpha-4200 \alpha^{2}+98 \alpha^{3} .
\end{aligned}\right.
$$




\section{Remark 3.3.}

- As it is was noted in [16], the stability condition on $\Delta t$ behaves as $C / \sqrt{\alpha}$ for $\alpha$ large enough. More precisely, $C=\sqrt{\frac{2}{(p+1)(p+2)}}$.

- This stability condition is constant for $\frac{p(p+1)}{2} \leq \alpha \leq \alpha_{1, p}$. This shows that it is not necessary to choose $\alpha$ too close from $\alpha_{p}^{0}$ to improve the CFL condition.

- In [2], Ainsworth et al. proved (3.1) for $p=0, \ldots, 3$ and conjectured this relation for any $p$. Theorem 3.2 extends its validity until $p=5$.

- The condition (3.1) does not depend on the dimension $d$. This would not have been the case if we had expressed $\gamma$ as a function of the circumcircle (or circumsphere) diameter which is $\sqrt{d} h$. Since $h$ is the diameter of the inscribed circle or sphere, we conjecture that the third definition of $\xi_{F}$ is the most appropriate. We will strengthen this conjecture when we discuss the extension of this theorem to meshes composed of rectangles or parallelepipeds.

We have performed numerical experiments to compute the numerical CFL condition on finite meshes. We have observed (see Sect. 6) that this condition was equivalent to condition (3.2), except for a small range of value of $\alpha$. Therefore we need a more restrictive necessary condition, which is provided by the following theorem.

Theorem 3.4. Let $V_{p, \alpha}=\left\{\lambda \in \mathbb{R}: Q_{p, \alpha}(\lambda)=0\right.$ and $\left.\left|\tilde{Q}_{p, \alpha}(\lambda)\right| \leq 1\right\}$ where $Q_{p, \alpha}(\lambda)$ is a polynomial of degree $2 p$ and $\tilde{Q}_{p, \alpha}(\lambda)$ is a rational function whose expressions are given in Appendix A. Let also $1 \leq p \leq 5$. Then, for the scheme (2.5) to be $L^{2}$-stable, the conditions (3.1), (3.2) and

$$
\left\{\begin{array}{l}
V_{p, \alpha}=\varnothing \\
o r \\
\sqrt{d} \frac{c \Delta t}{h} \leq C_{3, p}(\alpha)=\frac{1}{2 \sqrt{\max V_{p, \alpha}}}
\end{array}\right.
$$

have to be satisfied.

\section{Remark 3.5.}

- This theorem does not provide an explicit CFL condition. However, it can be computed numerically by the following algorithm:

1. Compute all the roots of $Q_{p, \alpha}$;

2. Select the real roots such that $\left|\tilde{Q}_{p, \alpha}(\lambda)\right| \leq 1$;

3. Choose the maximum of these roots.

- The numerical results in Section 6 show that this theorem gives in practical cases necessary and sufficient conditions.

- The numerical study of condition (3.3) that we present in Section 6 shows that the set $V_{p, \alpha}$ is actually empty except when $\alpha$ belongs to a small segment around $\alpha_{1}^{p}$. This means that Theorem 3.2 provides a sufficient and necessary stability condition when $\alpha$ is not in this segment. Moreover Remark 3.3 is still valid.

We were unfortunately unable to establish this theorem for any $p$ and we have restricted ourselves to $p \leq 5$. The proofs in the one dimensional case are given in Section 4 while the extension to $d=3$ is the subject of Section 5 . The proof for $d=2$ can be easily deduced from the case $d=3$. 


\section{Proof For the 1-Dimensional CASE}

This section contains the proofs of Theorems 3.2 and 3.4 in the one dimensional case. It consists of three steps. The first step is a Fourier analysis presented in Section 4.1; the second step is devoted to the proof of conditions (3.1) and is presented in Section 4.2; the last step concerning the proof of (3.2) and (3.3) is given in Section 4.3. The proofs are detailed for $p=3$ and are easily extendable to the cases $p=1,2,4$ and 5 .

Here, we assume that the domain is $\Omega=\mathbb{R}$ and is meshed by segments of length $h$. We consider a velocity $c^{2}=\mu / \rho=1$ but we can extend the proof to other velocities by setting $\Delta t^{\prime}=\Delta t / c$. We consider the scheme $(2.5)$ without source term that is to say

$$
M \frac{U^{n+1}-2 U^{n}+U^{n-1}}{\Delta t^{2}}+K U^{n}=0 .
$$

Considering the equation on one element $J$ of the mesh, we have $\forall J \in \mathcal{T}_{h}$

$$
M_{1, p} \frac{U_{J}^{n+1}-2 U_{J}^{n}+U_{J}^{n-1}}{\Delta t^{2}}+\left(K_{1, p}^{W}\right)^{T} U_{J-1}^{n}+K_{1, p} U_{J}^{n}+K_{1, p}^{W} U_{J+1}^{n}=0
$$

where $U_{J}$ corresponds to the vector of unknowns $U$ restricted to the element $J$ and $M_{1, p}, K_{1, p}$ and $K_{1, p}^{W}$ are respectively the mass and stiffness matrices in dimension 1 considering polynomials of degree $p$ :

$$
\begin{aligned}
M_{1, p}(i, j)= & h \int_{[0,1]} \hat{\varphi}_{i}(\hat{x}) \hat{\varphi}_{j}(\hat{x}) \mathrm{d} \hat{x}, \\
K_{1, p}(i, j)= & \frac{1}{h} \int_{[0,1]} \frac{\partial \hat{\varphi}_{i}}{\partial \hat{x}}(\hat{x}) \frac{\partial \hat{\varphi}_{j}}{\partial \hat{x}}(\hat{x}) \mathrm{d} \hat{x}+\frac{1}{2 h} \hat{\varphi}_{i}(1) \frac{\partial \hat{\varphi}_{j}}{\partial \hat{x}}(1)+\frac{1}{2 h} \hat{\varphi}_{j}(1) \frac{\partial \hat{\varphi}_{i}}{\partial \hat{x}}(1) \\
& +\gamma \hat{\varphi}_{i}(1) \hat{\varphi}_{j}(1)-\frac{1}{2 h} \hat{\varphi}_{i}(0) \frac{\partial \hat{\varphi}_{j}}{\partial \hat{x}}(0)-\frac{1}{2 h} \hat{\varphi}_{j}(0) \frac{\partial \hat{\varphi}_{i}}{\partial \hat{x}}(0) \\
& +\gamma \hat{\varphi}_{i}(0) \hat{\varphi}_{j}(0), \\
K_{1, p}^{W}(i, j)= & -\frac{1}{2 h} \hat{\varphi}_{i}(1) \frac{\partial \hat{\varphi}_{j}}{\partial \hat{x}}(0)+\frac{1}{2 h} \hat{\varphi}_{j}(0) \frac{\partial \hat{\varphi}_{i}}{\partial \hat{x}}(1)-\gamma \hat{\varphi}_{i}(1) \hat{\varphi}_{j}(0),
\end{aligned}
$$

where $\left\{\hat{\varphi}_{i}\right\}_{i=1, \ldots, p+1}$ are the classical discontinuous Lagrange basis functions on the reference element $[0,1]$.

\subsection{Fourier analysis of the IPDG scheme in 1D}

In order to study the stability of the IPDG scheme, we have to introduce the discrete Fourier transform

$$
\begin{aligned}
\mathcal{F}_{h}: L_{h}^{2} & \rightarrow L^{2}\left(K_{h}\right) \\
U & \rightarrow \tilde{U}=\mathcal{F}_{h}(U)(k)=\frac{h}{2 \pi} \sum_{J \in \mathbb{Z}} U_{J} \mathrm{e}^{-i k J h}
\end{aligned}
$$

with $K_{h}=\left[-\frac{\pi}{h}, \frac{\pi}{h}\right]$ and $L_{h}^{2}=\left\{U=\left(U_{J}\right)_{J \in \mathbb{Z}}, \sum_{J \in \mathbb{Z}}\left\|U_{J}\right\|^{2}<+\infty\right\}$.

Now, applying this discrete Fourier transform to (4.2), we obtain, $\forall \beta \in[-\pi, \pi]$

$$
M_{1, p} \frac{\tilde{U}_{J}^{n+1}(\beta)-2 \tilde{U}_{J}^{n}(\beta)+\tilde{U}_{J}^{n-1}(\beta)}{\Delta t^{2}}+K_{\beta} \tilde{U}_{J}^{n}(\beta)=0
$$

where $\beta=h k$ and $K_{\beta}=\left(K_{1, p}^{W}\right)^{T} \mathrm{e}^{-i \beta}+K_{1, p}+K_{1, p}^{W} \mathrm{e}^{i \beta}$.

The $L^{2}$-stability of (4.4) for all $\beta \in[-\pi, \pi]$, is equivalent to the $L^{2}$-stability of (4.1), thanks to the Parseval equalities. 
Since $M_{1, p}$ is positive definite and $K_{\beta}$ is Hermitian, all the eigenvalues of $N_{\beta}=M_{1, p}^{-1} K_{\beta}$ are real. Moreover, a classical stability analysis shows that (4.4) is stable if and only if

$$
0 \leq \lambda \leq \frac{4}{\Delta t^{2}}
$$

for all $\lambda \in \Lambda(\beta)$, where $\Lambda(\beta)$ denotes the set of the eigenvalues of $N_{\beta}$. Then, a necessary and sufficient condition for the stability of $(4.1)$ is

$$
\lambda_{\min } \geq 0 \quad \text { and } \quad \Delta t \leq \frac{2}{\sqrt{\lambda_{\max }}}
$$

with $\lambda_{\min }=\min _{\beta \in[-\pi, \pi]}[\min (\Lambda(\beta))]$ and $\lambda_{\max }=\max _{\beta \in[-\pi, \pi]}[\max (\Lambda(\beta))]$.

In Section 4.2, we show that the condition $\lambda_{\min } \geq 0$ is equivalent to (3.1) and in Section 4.3, we show that the condition $\lambda_{\max } \leq \frac{4}{\Delta t^{2}}$ implies (3.2) and (3.3).

\subsection{Proving the condition $\lambda_{\min } \geq 0$}

In the following, we consider the change of variable $\alpha=h \gamma$ to simplify the presentation.

To show the equivalence between (3.2) and $\lambda_{\min } \geq 0$, we have to consider the characteristic polynomial of $N_{\beta}$ :

$$
q_{\alpha}(\beta, \lambda)=(-1)^{p+1} \lambda^{p+1}+\sum_{i=0}^{p} c_{i}(\alpha, \beta) \lambda^{i} .
$$

The coefficients $c_{i}(\alpha, \beta)$ can be computed by a symbolic calculus software such as Maple. We present them in Appendix A for $1 \leq p \leq 5$.

In order to study the sign of the eigenvalues of $N_{\beta}$, we will use the following lemma.

Lemma 4.1. Let $P$ be a polynomial of degree $n$ with $n$ real roots such that $P(X)=\sum_{i=0}^{n} c_{i} X^{i}$. All the roots of $P$ are non negative if and only if

$$
(-1)^{i} c_{i} \geq 0
$$

Proof. The proof of this lemma can be found in [1].

Hence, we have to find a condition on $\alpha$ such that, $\forall i \in\{0, \ldots, p\}, \forall \beta \in[-\pi, \pi]$,

$$
(-1)^{i} c_{i}(\alpha, \beta) \geq 0
$$

Here we only detail the computations in the case $p=3$ and we give the expression of the characteristic polynomials for $p \neq 3$ in Appendix A. For $p=3$, we have

$$
\left\{\begin{array}{l}
c_{3}(\alpha, \beta)=\frac{8}{h^{2}}((15-\alpha) \cos (\beta)-4 \alpha) \\
c_{2}(\alpha, \beta)=\frac{240}{h^{4}}\left(\cos ^{2}(\beta)-(23+\alpha) \cos (\beta)+(18 \alpha-65)\right) \\
c_{1}(\alpha, \beta)=\frac{2880}{h^{6}}\left(4 \cos ^{2}(\beta)+(65-3 \alpha) \cos (\beta)+(141-32 \alpha)\right) \\
c_{0}(\alpha, \beta)=\frac{100800}{h^{8}}\left(3 \cos ^{2}(\beta)+2(3-\alpha) \cos (\beta)+(2 \alpha-9)\right) .
\end{array}\right.
$$


- Let us first study the condition on $c_{3}$. We have, $\forall \beta \in[-\pi, \pi]$,

$$
-c_{3}(\alpha, \beta) \geq 0 \Leftrightarrow(\alpha-15) \cos (\beta)+4 \alpha \geq 0 .
$$

It is clear that this condition is satisfied for all $\beta$ if and only if

$$
\left\{\begin{array}{l}
(\alpha-15)+4 \alpha \geq 0 \\
(15-\alpha)+4 \alpha \geq 0
\end{array}\right.
$$

which implies that

$$
\left\{\begin{array}{l}
\alpha \geq 3 \\
\alpha \geq-5
\end{array}\right.
$$

Consequently, $-c_{3}(\alpha, \beta) \geq 0$, whatever the choice of $\beta$, if and only if

$$
\alpha \geq 3
$$

- Let us now consider the condition on $c_{2}$.

Setting $X=\cos (\beta)$, this condition is equivalent to

$$
f_{\alpha}(X):=X^{2}-(23+\alpha) X+(18 \alpha-65) \geq 0, \forall X \in[-1,1]
$$

This second order polynomial admits two roots:

$$
\left\{\begin{array}{l}
X_{1}=\frac{1}{2}\left(23+\alpha+\left((\alpha-13)^{2}+620\right)^{1 / 2}\right), \\
X_{2}=\frac{1}{2}\left(23+\alpha-\left((\alpha-13)^{2}+620\right)^{1 / 2}\right) .
\end{array}\right.
$$

We know that $f_{\alpha}(X)$ is a second-order polynomial on the variable $X$ and its head coefficient is positive. Thus, to have $f_{\alpha}$ non negative $\forall X \in[-1 ; 1]$, we need one of the following conditions:

(1) the two roots are in $]-\infty ;-1]$ i.e. $X_{1} \leq-1$;

(2) the two roots are in $] 1 ;+\infty\left[\right.$ i.e. $X_{2} \geq 1$;

(3) $X_{1}=X_{2}$.

Since $(\alpha-13)^{2}+620>0, X_{1}>X_{2}$ and 3. is impossible.

The case $X_{1} \leq-1$ is also impossible since $X_{1} \geq 0$ when $\alpha \geq 0$ so we just have to consider the case $X_{2} \geq 1$, which leads to the inequality

which is equivalent to

$$
23+\alpha-\left((\alpha-13)^{2}+620\right)^{1 / 2} \geq 2
$$

$$
\alpha \geq \frac{87}{17}
$$

Finally, $c_{2}(\alpha, \beta) \geq 0$, whatever the choice of $\beta$, if and only if (4.9) holds.

- Now, let us study the sign of $c_{1}(\alpha, \beta)$.

Using the change of variable $X=\cos (\beta)$, the condition $-c_{1}(\alpha, \beta) \geq 0, \forall \beta \in[-\pi, \pi]$ is equivalent to

$$
f_{\alpha}(X):=-4 X^{2}+(3 \alpha-65) X+(32 \alpha-141) \geq 0, \forall X \in[-1,1] .
$$

The polynomial $f_{\alpha}$ admits the two following roots:

$$
\left\{\begin{array}{l}
X_{1}=\frac{-1}{8}\left(65-3 \alpha+\left(\left(3 \alpha+\frac{61}{3}\right)^{2}+\frac{14000}{9}\right)^{1 / 2}\right) \\
X_{2}=\frac{-1}{8}\left(65-3 \alpha-\left(\left(3 \alpha+\frac{61}{3}\right)^{2}+\frac{14000}{9}\right)^{1 / 2}\right) .
\end{array}\right.
$$


Since, the head coefficient of the polynom $f_{\alpha}$ is negative, we need $X_{1} \leq-1$ and $X_{2} \geq 1$.

The condition $X_{1} \leq-1$ implies that

$$
65-3 \alpha+\left(\left(3 \alpha+\frac{61}{3}\right)^{2}+\frac{14000}{9}\right)^{1 / 2} \geq 8
$$

which leads to

$$
\alpha \geq \frac{80}{29}
$$

In the same way, the condition $X_{2} \geq 1$ is equivalent to

$$
\alpha \geq 6 \text {. }
$$

Consequently, $-c_{1}(\alpha, \beta) \geq 0, \forall \beta \in[-\pi, \pi]$ if and only if $\alpha \geq 6$.

- Finally, let us look at the positivity of $c_{0}(\alpha, \beta), \forall \beta \in[-\pi, \pi]$.

Once again, using the change of variable $X=\cos (\beta)$, we have

$$
f_{\alpha}(X):=3 X^{2}+2(3-\alpha) X+2 \alpha-9 \geq 0 .
$$

This polynomial function $f_{\alpha}$ admits the two following roots:

$$
\left\{\begin{array}{l}
X_{1}=\frac{1}{3}(2 \alpha-9) \\
X_{2}=1
\end{array}\right.
$$

In the same way than previously, we need $X_{1} \geq 1$ which leads to the condition

$$
\alpha \geq 6 .
$$

In conclusion, taking into account the conditions (4.7), (4.9), (4.10), (4.11) and (4.12), we have

$$
\lambda_{\min } \geq 0 \Leftrightarrow \alpha \geq 6 .
$$

We used the same technique to derive a condition on $\alpha$ for all polynomial degrees $p$ from $p=1$ to $p=5$. Since the calculations are very similar, we do not detail them here and we just present the conditions in Table 1.

From these results, we can easily deduce the smallest penalization parameters ensuring the stability of the scheme (see Tab. 2). It is clear that, for $1 \leq p \leq 5$, the stability is guaranteed if and only if

$$
\alpha \geq \frac{p(p+1)}{2}, \text { or, equivalently, } \gamma \geq \frac{p(p+1)}{2 h} .
$$

\subsection{The CFL condition}

Now, we propose to prove that the condition

$$
\Delta t \leq \frac{2}{\sqrt{\lambda_{\max }}}
$$

implies (3.2) and (3.3). It is clear that for all $\beta \in[-\pi ; \pi]$, the polynomial $q_{\alpha}$ admits $p+1$ roots. We denote these roots, from the smallest to the greatest, $\lambda_{1}(\beta), \ldots, \lambda_{p+1}(\beta)$. Then, $\lambda_{\max }=\max _{\beta \in[-\pi, \pi]}[\max (\Lambda(\beta))]=$ $\max _{\beta \in[-\pi ; \pi]} \lambda_{p+1}(\beta)$. 
TABle 1. Conditions on $\alpha$ for each coefficient $c_{i}$ and each polynomial degree $p$.

\begin{tabular}{|c|c|c|c|c|c|c|}
\hline$p$ & $c_{0}$ & $c_{1}$ & $c_{2}$ & $c_{3}$ & $c_{4}$ & $c_{5}$ \\
\hline 1 & $\alpha \geq 1$ & $\alpha \geq 1$ & & & & \\
\hline 2 & $\alpha \geq 3$ & $\alpha \geq \frac{30}{11}$ & $\alpha \geq 2$ & & & \\
\hline 3 & $\alpha \geq 6$ & $\alpha \geq 6$ & $\alpha \geq \frac{87}{17}$ & $\alpha \geq 3$ & & \\
\hline 4 & $\alpha \geq 10$ & $\alpha \geq \frac{543}{55}$ & $\alpha \geq \frac{325}{34}$ & $\alpha \geq \frac{581}{73}$ & $\alpha \geq 4$ & \\
\hline 5 & $\alpha \geq 15$ & $\alpha \geq 15$ & $\alpha \geq \frac{336}{23}$ & $\alpha \geq \frac{1185}{86}$ & $\alpha \geq \frac{124}{11}$ & $\alpha \geq 5$ \\
\hline
\end{tabular}

TABLE 2. Stability condition on $\alpha$ for each polynomial degree $p$.

\begin{tabular}{|c|c|c|c|c|c|}
\hline$p$ & 1 & 2 & 3 & 4 & 5 \\
\hline & $\alpha \geq 1$ & $\alpha \geq 3$ & $\alpha \geq 6$ & $\alpha \geq 10$ & $\alpha \geq 15$ \\
\hline
\end{tabular}

The interval $[-\pi ; \pi]$ is a closed subspace of $\mathbb{R}$, then it exists $\beta_{\max }$ such that $\lambda_{\max }=\lambda_{p+1}\left(\beta_{\max }\right)$. Moreover, the real number $\beta_{\max }$ is such that one of the following conditions is satisfied:

(1) $\lambda_{p+1}^{\prime}\left(\beta_{\max }\right)=0$;

(2) $\beta_{\max }= \pm \pi$

(3) $\lambda_{p+1}$ is not differentiable in $\beta_{\max }$.

In order to exploit the conditions (1) and (3) we use the implicit function theorem.

The polynomial $q_{\alpha}$ satisfies $q_{\alpha}\left(\beta, \lambda_{p+1}(\beta)\right)=0$, then the implicit function theorem allows us to verify the existence of $\lambda_{p+1}^{\prime}(\beta)$ and to compute its value.

Thus, the conditions (1), (2) and (3) can be rewritten as:

(1) $\frac{\partial q_{\alpha}}{\partial \beta}\left(\beta_{\max }, \lambda_{p+1}\left(\beta_{\max }\right)\right)=0 ;$
(2) $\beta_{\max }= \pm \pi ;$
(3) $\frac{\partial q_{\alpha}}{\partial \lambda}\left(\beta_{\max }, \lambda_{p+1}\left(\beta_{\max }\right)\right)=0$.

Consequently, we have to determine all the $\beta$ satisfying (1), (2) or (3). Then, we have

$$
\lambda_{\max }=\max _{\beta \in \mathcal{A}} \lambda_{p+1}(\beta)
$$

where $\mathcal{A}=\{\beta \in[-\pi ; \pi]$ such that one of the conditions (1), (2) or (3) is satisfied $\}$.

However, the third condition gives no usable information. We restrict ourselves to $\beta \in \tilde{\mathcal{A}}$ where $\tilde{\mathcal{A}}=$ $\{\beta \in[-\pi ; \pi]$ : such that one of the conditions (1) or (2) is satisfied $\}$.

Then, we have $\lambda_{\max } \geq \max _{\beta \in \tilde{\mathcal{A}}} \lambda_{p+1}(\beta)$ which means that the stability condition we will obtain will just be necessary.

In the following, we only detail the case $p=3$, since the proofs are similar for all $p=1, \ldots, 5$. 
For the condition (1), we are seeking $\left(\beta_{0}, \lambda\left(\beta_{0}\right)\right)$ such that $\frac{\partial q_{\alpha}}{\partial \beta}\left(\beta_{0}, \lambda\left(\beta_{0}\right)\right)=0$. Since

$$
\begin{aligned}
\frac{\partial q_{\alpha}}{\partial \beta}\left(\beta_{0}, \lambda\left(\beta_{0}\right)\right)= & \sin \left(\beta_{0}\right)\left[\frac{8}{h^{2}}(\alpha-15) \lambda^{3}\left(\beta_{0}\right)+\frac{240}{h^{4}}\left(23+\alpha-2 \cos \left(\beta_{0}\right)\right) \lambda^{2}\left(\beta_{0}\right)\right. \\
& \left.+\frac{2880}{h^{6}}\left(3 \alpha-65-8 \cos \left(\beta_{0}\right)\right) \lambda\left(\beta_{0}\right)+\frac{100800}{h^{8}}\left(-6 \cos \left(\beta_{0}\right)+2(\alpha-3)\right)\right] \\
:= & \sin \left(\beta_{0}\right) \tilde{q}_{\alpha}\left(\beta_{0}, \lambda\left(\beta_{0}\right)\right) .
\end{aligned}
$$

we obtain the two following conditions

$$
\frac{\partial q_{\alpha}}{\partial \beta}\left(\beta_{0}, \lambda\left(\beta_{0}\right)\right)=0 \Leftrightarrow\left\{\begin{array}{l}
\sin \left(\beta_{0}\right)=0 \\
\text { or } \\
\tilde{q}_{\alpha}\left(\beta_{0}, \lambda\left(\beta_{0}\right)\right)=0 .
\end{array}\right.
$$

First, we consider only the condition $\sin \left(\beta_{0}\right)=0$.

- If $\beta_{0}=0$, we obtain the following eigenvalues:

$$
0 ; \frac{60}{h^{2}} ; \frac{90+20 \alpha+2 g_{1}(\alpha)}{h^{2}} ; \frac{90+20 \alpha-2 g_{1}(\alpha)}{h^{2}}
$$

where $g_{1}(\alpha)=\left(4545-1320 \alpha+100 \alpha^{2}\right)^{\frac{1}{2}}$.

It is clear that, for $\alpha \geq 0$, the two greatest eigenvalues are $x_{1}=\frac{60}{h^{2}}$ and $x_{2}=\frac{1}{h^{2}}\left(90+20 \alpha+2 g_{1}(\alpha)\right)$. Studying the sign of the quantity

$$
h^{2}\left(x_{1}-x_{2}\right)=-150+20 \alpha+2 g_{1}(\alpha)
$$

we can easily obtain

$$
\left\{\begin{array}{lll}
\lambda_{4}(0)=x_{2} & \text { if } & \alpha \geq 6 \\
\lambda_{4}(0)=x_{1} & \text { if } & \alpha<6 .
\end{array}\right.
$$

We have proved in Section 4.2 that the condition $\alpha \geq 6$ is a necessary stability condition. Therefore, we only have to consider $\lambda_{4}=x_{2}$.

- If $\beta_{0}= \pm \pi$, we obtain the following eigenvalues:

$$
\frac{2}{h^{2}}(45+\sqrt{1605}) ; \frac{2}{h^{2}}(45-\sqrt{1605}) ; \frac{2}{h^{2}}\left(-15+6 \alpha+g_{2}(\alpha)\right) ; \frac{2}{h^{2}}\left(-15+6 \alpha-g_{2}(\alpha)\right)
$$

where $g_{2}(\alpha)=\left(405-240 \alpha+36 \alpha^{2}\right)^{\frac{1}{2}}$.

The two greatest eigenvalues are $x_{3}=\frac{2}{h^{2}}(45+\sqrt{1605})$ and $x_{4}=\frac{2}{h^{2}}\left(-15+6 \alpha+g_{2}(\alpha)\right)$. The study of the sign of $x_{3}-x_{4}$ implies

$$
\left\{\begin{array}{l}
\lambda_{4}(\pi)=x_{3} \text { if } \alpha \leq 10 \\
\lambda_{4}(\pi)=x_{4} \text { if } \alpha \geq 10 .
\end{array}\right.
$$

Now we have to compare $\lambda_{4}(0)$ and $\lambda_{4}(\pi)$. We easily verify that

$$
\left\{\begin{array}{l}
\lambda_{4}(0) \geq \lambda_{4}(\pi) \text { if } \alpha \geq \frac{2 \sqrt{1605}+393}{49} \simeq 9.66, \\
\lambda_{4}(\pi)>\lambda_{4}(0) \text { if } \frac{2 \sqrt{1605}+393}{49}<\alpha .
\end{array}\right.
$$

Then, we have $\max _{\beta \in \tilde{\mathcal{A}}} \lambda_{4}(\beta) \geq \max _{\beta=\{0, \pi\}} \lambda_{4}(\beta)$. 
Consequently, considering (4.15) and (4.17), a necessary condition of stability is

$$
\frac{\Delta t}{h} \leq\left\{\begin{array}{l}
\frac{2}{\sqrt{\lambda_{4}(\pi)}} \text { if } 6 \leq \alpha \leq \alpha_{1, p}, \\
\frac{2}{\sqrt{\lambda_{4}(0)}} \text { if } \alpha_{1, p}<\alpha,
\end{array}\right.
$$

with $\alpha_{1, p}=\frac{2 \sqrt{1605}+393}{49}$, which corresponds to the necessary condition (3.1). We remark that the condition $\sin \left(\beta_{0}\right)=0$ implies $(2)$ then we do not need to consider this condition once again.

Let us now find $\left(\beta_{0}, \lambda\left(\beta_{0}\right)\right)$ such that $\tilde{q}_{\alpha}\left(\beta_{0}, \lambda\left(\beta_{0}\right)\right)=0$. In fact, we don't have to compute $\beta_{0}$, we interest ourselves only to $\max \Lambda\left(\beta_{0}\right)$.

We can easily obtain that

$\cos \left(\beta_{0}\right)=\frac{(\alpha-15) h^{6} \lambda^{3}\left(\beta_{0}\right)+30(23+\alpha) h^{4} \lambda^{2}\left(\beta_{0}\right)+360(3 \alpha-65) h^{2} \lambda\left(\beta_{0}\right)+25200(\alpha-3)}{60\left(h^{4} \lambda^{2}\left(\beta_{0}\right)+48 h^{2} \lambda\left(\beta_{0}\right)+1260\right)}:=\tilde{Q}_{3, \alpha}\left(\lambda\left(\beta_{0}\right)\right)$.

Using this expression of $\cos \left(\beta_{0}\right)$ in the characteristic polynomial $q_{\alpha}$ we obtain that $\lambda\left(\beta_{0}\right)$ is solution to

$$
q_{\alpha}\left(\beta_{0}, \lambda\left(\beta_{0}\right)\right)=-\frac{1}{15 h^{8}\left(h^{4} \lambda^{2}\left(\beta_{0}\right)+48 h^{2} \lambda\left(\beta_{0}\right)+1260\right)} \sum_{i=0}^{6} \lambda^{i}\left(\beta_{0}\right) h^{2 i} \tilde{c}_{i}(\alpha)=0
$$

or, equivalently, solution to

$$
Q_{3, \alpha}\left(\lambda\left(\beta_{0}\right)\right)=\sum_{i=0}^{6} \lambda^{i}\left(\beta_{0}\right) h^{2 i} \tilde{c}_{i}(\alpha)=0
$$

with

$$
\left\{\begin{array}{l}
\tilde{c}_{0}(\alpha)=635040000\left(\alpha^{2}-12 \alpha+36\right) \\
\tilde{c}_{1}(\alpha)=3628800\left(15 \alpha^{2}+70 \alpha-96\right), \\
\tilde{c}_{2}(\alpha)=86400\left(31 \alpha^{2}-447 \alpha+5316\right), \\
\tilde{c}_{3}(\alpha)=14400\left(8 \alpha^{2}-135 \alpha-1728\right) \\
\tilde{c}_{4}(\alpha)=180\left(17 \alpha^{2}-442 \alpha+7740\right) \\
\tilde{c}_{5}(\alpha)=60\left(\alpha^{2}+16 \alpha-357\right) \\
\tilde{c}_{6}(\alpha)=\alpha^{2}-30 \alpha+210 .
\end{array}\right.
$$

After having computed the roots of $Q_{3, \alpha}$, we have to verify that $\beta_{0}$ is well defined, that is to say $\left|\tilde{Q}_{3, \alpha}(\lambda)\right| \leq 1$. That is why we are interested uniquely in the eigenvalues $\lambda$ verifying $Q_{3, \alpha}(\lambda)=0$ and $\left|\tilde{Q}_{3, \alpha}(\lambda)\right| \leq 1$, that is to say in the elements of $V_{3, \alpha}$.

Finally, we have $\max _{\beta \in \tilde{\mathcal{A}}} \lambda_{4}(\beta)=\max \left[\max \left[\lambda_{4}(0), \lambda_{4}(\pi)\right]\right.$, $\left.\max V_{3, \alpha}\right]$. We can easily deduce the results of Theorem 3.4. 


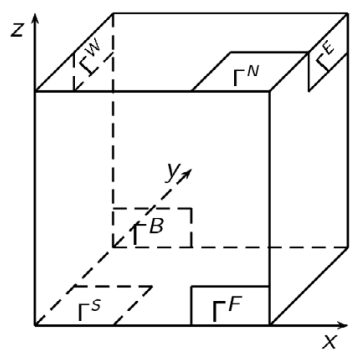

Figure 1. Notations of the faces in 3D.

\section{The $d$-Dimensional CASE}

In this section, we propose to adapt the technique proposed in [13] to extend the analysis from the 1D case to the $d \mathrm{D}$ case. Here, we only detail the three-dimensional case, since the technique is exactly the same for the two dimensional case.

First of all, we consider an infinite homogeneous 3D domain $\Omega$ uniformly meshed by cubes of edge $h$.

We introduce the following notations.

- $\Omega=\bigcup_{K_{J} \in \mathcal{T}_{h}} K_{J}$ where $K_{J}=\prod_{k=1}^{3} S_{J_{k}}=\prod_{k=1}^{3}\left[J_{k} h,\left(J_{k}+1\right) h\right]$ and $J=\left(J_{k}\right)_{k=1, \ldots, 3}$.

- On $\hat{K}[0,1]^{d}$, we define the Lagrange basis functions $\left(\hat{\varphi}_{1}\right)_{1 \in\{1, \ldots, p+1\}^{3}}$ by

$$
\hat{\varphi}_{\mathbf{l}}(\mathbf{x})=\prod_{k=1}^{3} \hat{\varphi}_{l_{k}}\left(x_{k}\right)
$$

where $\hat{\varphi}_{l_{k}}$ are the 1D Lagrange basis functions.

- Since the mesh is uniform, the basis functions are defined thanks to the functions $\left(\hat{\varphi}_{1}\right)_{1 \in\{1, \ldots, p+1\}^{3}}$ by

$$
\varphi_{\mathbf{m}}^{J}(\mathbf{x})=\hat{\varphi}_{\mathbf{m}}\left(\frac{x-J h}{h}\right) \mathbb{1}_{K_{\mathbf{j}}}(\mathbf{x})
$$

where $\mathbb{1}_{K_{J}}$ is the indicator function of $K_{J}$. These functions can be written as a product of $d 1 \mathrm{D}$ basis functions:

$$
\varphi_{\mathbf{m}}^{J}(\mathbf{x})=\prod_{k=1}^{3} \hat{\varphi}_{m_{k}}\left(\frac{x-J_{k} h}{h}\right) \mathbb{1}_{S_{J_{k}}}\left(\mathbf{x}_{k}\right) .
$$

- The different faces of the reference element $\hat{K}$ are denoted by an exponent $C$ corresponding to the orientation of the face: North (N), South (S), East (E), West (W), in Front of (F) and in the Back (B) (cf. Fig. 1).

Since the mesh is uniform, we can rewrite the problem on an element $\mathbf{I}=\left\{I_{1}, I_{2}, I_{3}\right\}$ :

$$
\begin{aligned}
M_{3, p} \delta^{n} U_{I_{1}, I_{2}, I_{3}}= & K_{3, p} U_{I_{1}, I_{2}, I_{3}}+K_{3, p}^{E} U_{I_{1}+1, I_{2}, I_{3}}+K_{3, p}^{W} U_{I_{1}-1, I_{2}, I_{3}} \\
& +K_{3, p}^{F} U_{I_{1}, I_{2}+1, I_{3}}+K_{3, p}^{B} U_{I_{1}, I_{2}-1, I_{3}}+K_{3, p}^{N} U_{I_{1}, I_{2}, I_{3}+1} \\
& +K_{3, p}^{S} U_{I_{1}, I_{2}, I_{3}-1}
\end{aligned}
$$


where

- $U_{I_{1}, I_{2}, I_{3}}$ corresponds to the restriction of $U$ on the element $I$;

- $\delta^{n} U_{I_{1}, I_{2}, I_{3}}=\frac{U_{I_{1}, I_{2}, I_{3}}^{n+1}-2 U_{I_{1}, I_{2}, I_{3}}^{n}+U_{I_{1}, I_{2}, I_{3}}^{n-1}}{\Delta t^{2}}$;

- $M_{3, p}$ is a block of the mass matrix $M$;

$$
M_{3, p}(\mathbf{i}, \mathbf{j})=h^{3} \int_{\hat{K}} \hat{\varphi}_{\mathbf{i}} \hat{\varphi}_{\mathbf{j}} \mathrm{d} \hat{x}, \quad \mathbf{i}, \mathbf{j} \in\{1, \ldots, p+1\}^{3} ;
$$

- $K_{3, p}$ is a diagonal block of the matrix $K$

$$
\begin{aligned}
K_{3, p}(\mathbf{i}, \mathbf{j})= & h \int_{\hat{K}} \nabla \hat{\varphi}_{\mathbf{i}} \cdot \nabla \hat{\varphi}_{\mathbf{j}} \mathrm{d} \hat{x}-\sum_{C \in\{N, S, E, W, B, F\}} \frac{h}{2} \int_{\Gamma^{C}}\left(\hat{\varphi}_{\mathbf{i}} \nabla \hat{\varphi}_{\mathbf{j}}+\hat{\varphi}_{\mathbf{j}} \nabla \hat{\varphi}_{\mathbf{i}}\right) \nu_{C} \mathrm{~d} \sigma \\
& +\sum_{C \in\{N, S, E, W, B, F\}} h^{2} \int_{\Gamma^{C}} \gamma \hat{\varphi}_{\mathbf{i}} \hat{\varphi}_{\mathbf{j}} \mathrm{d} \sigma, \quad \mathbf{i}, \mathbf{j} \in\{1, \ldots, p+1\}^{3}
\end{aligned}
$$

where $\nu_{C}$ is the outward unit normal vector to the face $\Gamma^{C}$.

- $K_{3, p}^{C}$ is a block of the matrix $K$ corresponding to the interactions between an element $I$ and its neighbour on the face $\Gamma^{C}$ :

$$
\begin{aligned}
K_{3, p}^{E}(\mathbf{i}, \mathbf{j})= & \int_{[0,1]^{2}} \frac{h}{2}\left(\hat{\varphi}_{\mathbf{i}}\left(1, x_{2}, x_{3}\right) \nabla \hat{\varphi}_{\mathbf{j}}\left(0, x_{2}, x_{3}\right)+\hat{\varphi}_{\mathbf{j}}\left(0, x_{2}, x_{3}\right) \nabla \hat{\varphi}_{\mathbf{i}}\left(1, x_{2}, x_{3}\right)\right) \nu_{E} \\
& -h^{2} \hat{\varphi}_{\mathbf{i}}\left(1, x_{2}, x_{3}\right) \hat{\varphi}_{\mathbf{j}}\left(0, x_{2}, x_{3}\right) \mathrm{d} x_{2} \mathrm{~d} x_{3} \\
K_{3, p}^{F}(\mathbf{i}, \mathbf{j})= & \int_{[0,1]^{2}} \frac{h}{2}\left(\hat{\varphi}_{\mathbf{i}}\left(x_{1}, 1, x_{3}\right) \nabla \hat{\varphi}_{\mathbf{j}}\left(x_{1}, 0, x_{3}\right)+\hat{\varphi}_{\mathbf{j}}\left(x_{1}, 0, x_{3}\right) \nabla \hat{\varphi}_{\mathbf{i}}\left(x_{1}, 1, x_{3}\right)\right) \nu_{F} \\
& -h^{2} \hat{\varphi}_{\mathbf{i}}\left(x_{1}, 1, x_{3}\right) \hat{\varphi}_{\mathbf{j}}\left(x_{1}, 0, x_{3}\right) \mathrm{d} x_{1} \mathrm{~d} x_{3} \\
K_{3, p}^{N}(\mathbf{i}, \mathbf{j})= & \int_{[0,1]^{2}} \frac{h}{2}\left(\hat{\varphi}_{\mathbf{i}}\left(x_{1}, x_{2}, 1\right) \nabla \hat{\varphi}_{\mathbf{j}}\left(x_{1}, x_{2}, 0\right)+\hat{\varphi}_{\mathbf{j}}\left(x_{1}, x_{2}, 0\right) \nabla \hat{\varphi}_{\mathbf{i}}\left(x_{1}, x_{2}, 1\right)\right) \nu_{N} \\
& -h^{2} \hat{\varphi}_{\mathbf{i}}\left(x_{1}, x_{2}, 1\right) \hat{\varphi}_{\mathbf{j}}\left(x_{1}, x_{2}, 0\right) \mathrm{d} x_{1} \mathrm{~d} x_{2} \\
& K_{3, p}^{W}\left(\left(i_{1}, i_{2}, i_{3}\right),\left(j_{1}, j_{2}, j_{3}\right)\right)=K_{3, p}^{E}\left(\left(j_{1}, i_{2}, i_{3}\right),\left(i_{1}, j_{2}, j_{3}\right)\right) \\
& K_{3, p}^{B}\left(\left(i_{1}, i_{2}, i_{3}\right),\left(j_{1}, j_{2}, j_{3}\right)\right)=K_{3, p}^{F}\left(\left(i_{1}, j_{2}, i_{3}\right),\left(j_{1}, i_{2}, j_{3}\right)\right) \\
& K_{3, p}^{S}\left(\left(i_{1}, i_{2}, i_{3}\right),\left(j_{1}, j_{2}, j_{3}\right)\right)=K_{3, p}^{N}\left(\left(i_{1}, i_{2}, j_{3}\right),\left(j_{1}, j_{2}, i_{3}\right)\right) .
\end{aligned}
$$

Then, multiplying equation (5.1) by the inverse of the mass matrix $M_{3, p}$, we obtain

$$
\begin{aligned}
\delta^{n} U_{I_{1}, I_{2}, I_{3}}= & N_{3, p} U_{I_{1}, I_{2}, I_{3}}+N_{3, p}^{E} U_{I_{1}+1, I_{2}, I_{3}}+N_{3, p}^{W} U_{I_{1}-1, I_{2}, I_{3}} \\
& +N_{3, p}^{F} U_{I_{1}, I_{2}+1, I_{3}}+N_{3, p}^{B} U_{I_{1}, I_{2}-1, I_{3}}+N_{3, p}^{N} U_{I_{1}, I_{2}, I_{3}+1} \\
& +N_{3, p}^{S} U_{I_{1}, I_{2}, I_{3}-1}
\end{aligned}
$$

where $N_{3, p}=M_{3, p}^{-1} K_{3, p}$ and $N_{3, p}^{C}=M_{3, p}^{-1} K_{3, p}^{C}$.

Now, we are interested in rewriting the matrices $N_{3, p}$ and $N_{3, p}^{C}$ with respect to the matrices we have obtained for the one dimensional case. 


\subsection{From the 3 dimensional case to the one dimensional case}

The coefficients of $M_{3, p}, K_{3, p}, K_{3, p}^{C}, N_{3, p}$ and $N_{3, p}^{C}$ can be deduced from the coefficients of $M_{1, p}, K_{1, p}, K_{1, p}^{W}$, $N_{1, p}$ and $N_{1, p}^{W}$ thanks to the following theorem.

Theorem 5.1. For all $\mathbf{m}=\left(m_{k}\right)_{k=1, \ldots, 3} \in\{1, \ldots, p+1\}^{3}$ and $\mathbf{n}=\left(n_{k}\right)_{k=1, \ldots, 3} \in\{1, \ldots, p+1\}^{3}$, we have

$$
\begin{aligned}
& \text { 1. } M_{3, p}(\mathbf{m}, \mathbf{n})=\prod_{i=1}^{3} M_{1, p}\left(m_{i}, n_{i}\right), \\
& \text { 2. } K_{3, p}(\mathbf{m}, \mathbf{n})=\sum_{i=1}^{3}\left(K_{1, p}\left(m_{i}, n_{i}\right) \prod_{k=1, k \neq i}^{3} M_{1, p}\left(m_{k}, n_{k}\right)\right) \text {, } \\
& \text { 3. } K_{3, p}^{C}(\mathbf{m}, \mathbf{n})=K_{1, p}^{W}\left(m_{p_{C}}, n_{p_{C}}\right) \prod_{k=1, k \neq p_{C}}^{3} M_{1, p}\left(m_{k}, n_{k}\right), \\
& \text { 4. } N_{3, p}(\mathbf{m}, \mathbf{n})=\sum_{p=1}^{3}\left(N_{1, p}\left(m_{p}, n_{p}\right) \prod_{k=1, k \neq p}^{3} \delta_{m_{k}, n_{k}}\right), \\
& \text { 5. } N_{3, p}^{C}(\mathbf{m}, \mathbf{n})=N_{1, p}^{W}\left(m_{p_{C}}, n_{p_{C}}\right) \prod_{k=1, k \neq p_{C}}^{3} \delta_{m_{k}, n_{k}},
\end{aligned}
$$

where $p_{C}=\left\{\begin{array}{l}1 \text { if } C \in\{E, W\}, \\ 2 \text { if } C \in\{N, S\}, \quad \text { and } N_{1, p}=M_{1, p}^{-1} K_{1, p} \text { and } N_{1, p}^{C}=M_{1, p}^{-1} K_{1, p}^{C} . \\ 3 \text { if } C \in\{B, F\},\end{array}\right.$

The proof of this theorem is given in Appendix C.

\subsection{Consequences of the stability analysis}

Let us now apply a Fourier transform in the three directions to (5.2) to obtain, for $\beta=[-\pi, \pi]^{3}$,

$$
\delta^{n} \tilde{U}_{\beta_{1}, \beta_{2}, \beta_{3}}=\mathbf{N}_{\beta} \tilde{U}_{\beta_{1}, \beta_{2}, \beta_{3}}
$$

where the matrix $\mathbf{N}_{\boldsymbol{\beta}}$ is defined by

$$
\begin{aligned}
& \mathbf{N}_{\boldsymbol{\beta}}(\mathbf{m}, \mathbf{n})= \\
& \sum_{j=1}^{3}\left[N_{1, p}\left(m_{j}, n_{j}\right) \prod_{q=1, q \neq j}^{3} \delta_{m_{q}, n_{q}}+\mathrm{e}^{i \beta_{j}} N_{1, p}^{W}\left(m_{j}, n_{j}\right) \prod_{q=1, q \neq j}^{3} \delta_{m_{q}, n_{q}}+\mathrm{e}^{-i \beta_{j}} N_{1, p}^{W}\left(n_{j}, m_{j}\right) \prod_{q=1, q \neq j}^{3} \delta_{m_{q}, n_{q}}\right]
\end{aligned}
$$

which can be rewritten as

$$
\begin{aligned}
\mathbf{N}_{\boldsymbol{\beta}}(\mathbf{m}, \mathbf{n}) & =\sum_{j=1}^{3}\left(\left(N_{1, p}\left(m_{j}, n_{j}\right)+\mathrm{e}^{i \beta_{j}} N_{1, p}^{W}\left(m_{j}, n_{j}\right)+\mathrm{e}^{-i \beta_{j}} N_{1, p}^{W}\left(n_{j}, m_{j}\right)\right) \prod_{q=1, q \neq j}^{3} \delta_{m_{q}, n_{q}}\right) \\
& =\sum_{j=1}^{3} N_{\beta_{j}}\left(m_{j}, n_{j}\right) \prod_{q=1, q \neq j}^{3} \delta_{m_{q}, n_{q}} .
\end{aligned}
$$

Using the stability analysis as in Section 4.1 , the stability of the scheme is ensured if and only if

$$
\lambda_{\min , 3} \geq 0 \quad \text { and } \quad \lambda_{\max , 3} \leq \frac{4}{\Delta t^{2}}
$$


where $\lambda_{\min , 3}=\min _{\boldsymbol{\beta} \in[-\pi, \pi]^{3}}\left(\min \Lambda\left(\mathbf{N}_{\boldsymbol{\beta}}\right)\right), \lambda_{\max , 3}=\max _{\boldsymbol{\beta} \in[-\pi, \pi]^{3}}\left(\max \Lambda\left(\mathbf{N}_{\boldsymbol{\beta}}\right)\right)$ and $\Lambda\left(\mathbf{N}_{\boldsymbol{\beta}}\right)$ is the set of eigenvalues of $\mathbf{N}_{\beta}$.

To compute these values, we use the following lemma.

Lemma 5.2. Let $\left(\lambda_{i}^{\boldsymbol{\beta}}\right)_{i=1, \ldots, p+1}$ denote the eigenvalues of $N_{\boldsymbol{\beta}}$ and $\left(v_{i}^{\boldsymbol{\beta}}\right)_{i=1, \ldots, p+1}$ the associated eigenvectors. Then, the eigenvalues of $\mathbf{N}_{\boldsymbol{\beta}}$ are given by

$$
\lambda_{\mathbf{i}}^{\boldsymbol{\beta}}=\sum_{k=1}^{3} \lambda_{i_{k}}^{\beta_{k}}
$$

and the associated eigenvectors by

$$
v_{\mathbf{i}}^{\boldsymbol{\beta}}(\mathbf{m})=\prod_{k=1}^{3} v_{i_{k}}^{\beta_{k}}\left(m_{k}\right) .
$$

Proof. Let $v_{\mathbf{i}}^{\boldsymbol{\beta}}$ defined by (5.5), then

$$
\begin{aligned}
\left(N_{\boldsymbol{\beta}} v_{\mathbf{i}}^{\boldsymbol{\beta}}\right)(\mathbf{m}) & =\sum_{n_{1}, \ldots, n_{3}=1}^{p+1}\left(\sum_{q=1}^{3} N_{\beta_{q}}\left(m_{q}, n_{q}\right) \prod_{k=1, k \neq q}^{3} \delta_{m_{k}, n_{k}} \prod_{k=1}^{3} v_{i_{k}}^{\beta_{k}}\left(n_{k}\right)\right) \\
& =\sum_{q=1}^{3}\left(\left(\sum_{n_{q}=1}^{p+1} N_{\beta_{q}}\left(m_{q}, n_{q}\right) v_{i_{q}}^{\beta_{q}}\left(n_{q}\right)\right) \prod_{k=1, k \neq q}^{3} v_{i_{k}}^{\beta_{k}}\left(m_{k}\right)\right) \\
& =\sum_{q=1}^{3}\left(\lambda_{i_{q}}^{\beta_{q}} v_{i_{q}}^{\beta_{q}}\left(m_{q}\right) \prod_{k=1, k \neq q}^{3} v_{i_{k}}^{\beta_{k}}\left(m_{k}\right)\right) \\
& =\left(\sum_{q=1}^{3} \lambda_{i_{q}}^{\beta_{q}}\right) v_{\mathbf{i}}^{\boldsymbol{\beta}}(\mathbf{m})
\end{aligned}
$$

It is then clear that

$$
\lambda_{\min , 3}=3 \lambda_{\min } \quad \text { and } \quad \lambda_{\max , 3}=3 \lambda_{\max } .
$$

Hence, the scheme (5.5) is stable, if and only if

$$
\lambda_{\min } \geq 0 \quad \text { and } \quad \frac{c \Delta t}{h} \leq \frac{1}{\sqrt{3}} \sqrt{\frac{2}{\lambda_{\max }}} .
$$

The first condition is equivalent to condition (3.1), while the second one implies (3.2) and (3.3).

\subsection{Extension to rectangular or parallelepiped mesh}

For the sake of simplicity, we restricted our theorem to mesh consisting of squares or cubes. However, one can extend the proof to the case of rectangular or parallelepipeds meshes to show that a necessary stability condition is in $2 \mathrm{D}$ :

$$
\gamma \geq \frac{p(p+1)}{2 \min \left(h_{x}, h_{y}\right)}
$$

and in 3D:

$$
\gamma \geq \frac{p(p+1)}{2 \min \left(h_{x}, h_{y}, h_{z}\right)} .
$$

Here, $h_{x}, h_{y}$ and $h_{z}$ denote respectively the length of the edges of the elements in the $x, y$ and $z$ direction. The minimal value of $h_{x}, h_{y}$ and $h_{z}$ is actually the diameter of the inscribed sphere of each element. This remark 


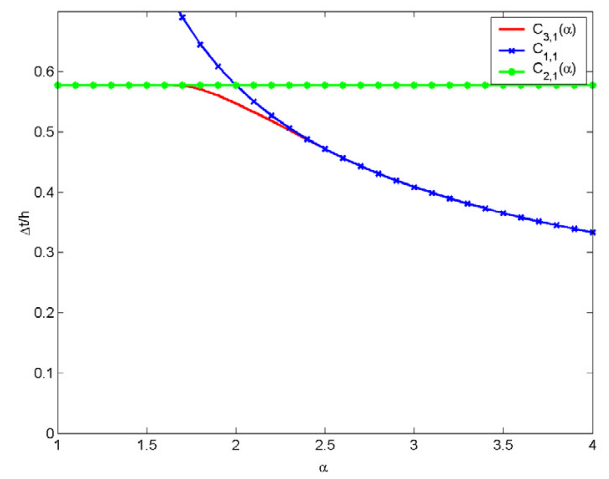

Figure 2. The 3 conditions for $p=1$.

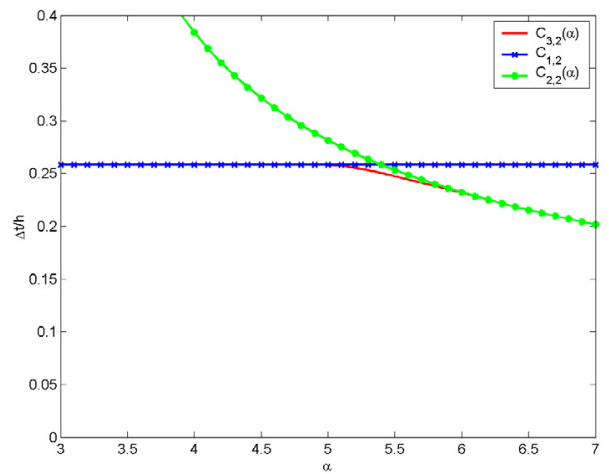

Figure 4 . The 3 conditions for $p=2$.

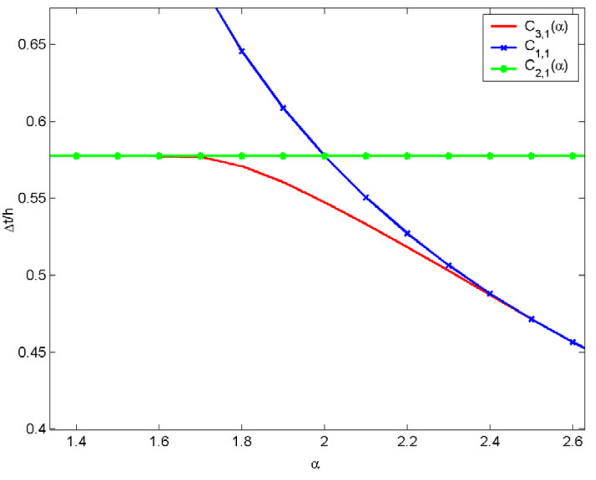

Figure 3 . Zoom on the 3 cond. for $p=1$.

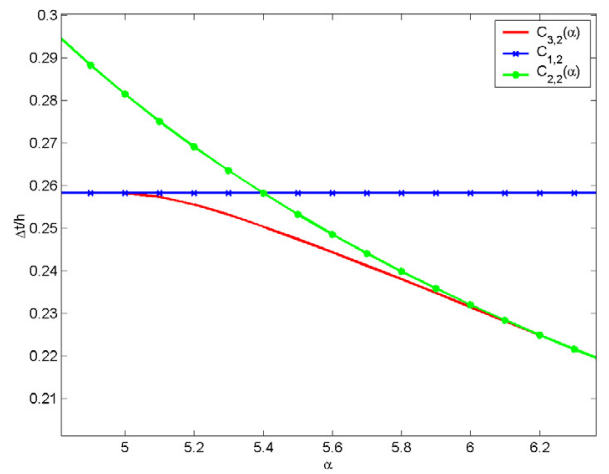

Figure 5. Zoom on the 3 cond. for $p=2$.

confirms that the third definition of $\xi_{F}$ using the diameter of the inscribed sphere or circle in 2D is the most appropriate.

The proof could also be extended to obtain a CFL condition, but its expression is complicated and does not add much insight.

\section{NumericAl RESULtS}

In this section, we first represent the behaviour of the CFL condition with respect to $\alpha$ and we show that the set $V_{p, \alpha}$ is empty for almost all the values of $\alpha$ (Sect. 6.1). This illustrate the fact that Theorem 3.2 is actually necessary and sufficient for most of the values of $\alpha$. Then, we compare our analytical CFL condition in infinite domain to the CFL condition computed numerically on finite meshes in order to illustrate the validity of Theorem 3.4 (Sect. 6.2). Indeed, one of the main goal of this part is to illustrate the fact that the theoretical results we have obtained are valid for various kind of boundary conditions.

\subsection{Behaviour of the CFL condition with respect to $\alpha$}

In Figures 2, 4, 6, 8 and 10 we plot the functions $C_{1, p}$ (blue line with diamonds), $C_{2, p}(\alpha)$ (red line with circles) and $C_{3, p}(\alpha)$ (black line) respectively for $p=1,2,3,4$ and 5 . The function $C_{3, p}(\alpha)$ only modifies the 


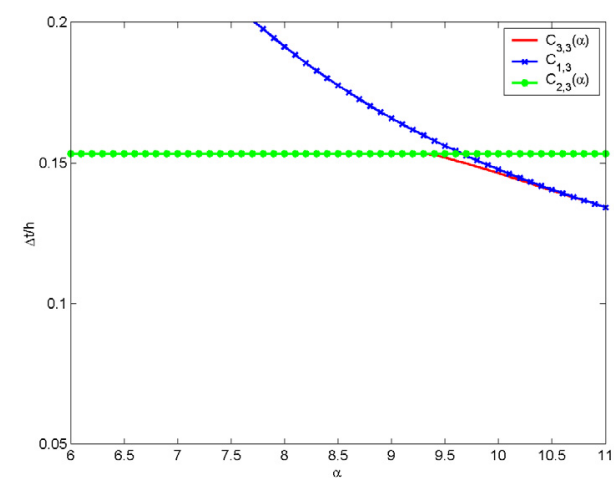

Figure 6 . The 3 conditions for $p=3$.

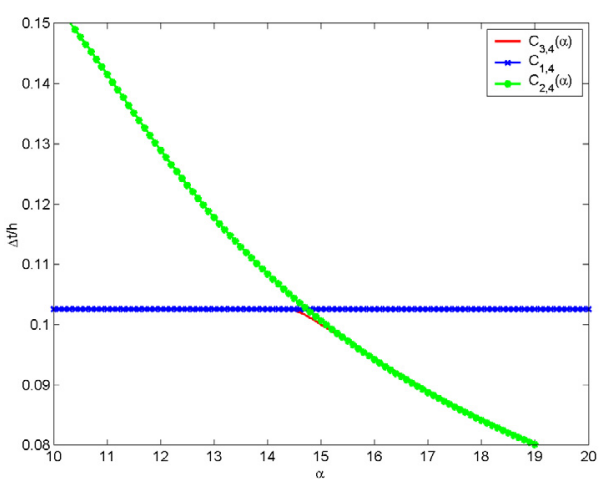

Figure 8 . The 3 conditions for $p=4$.

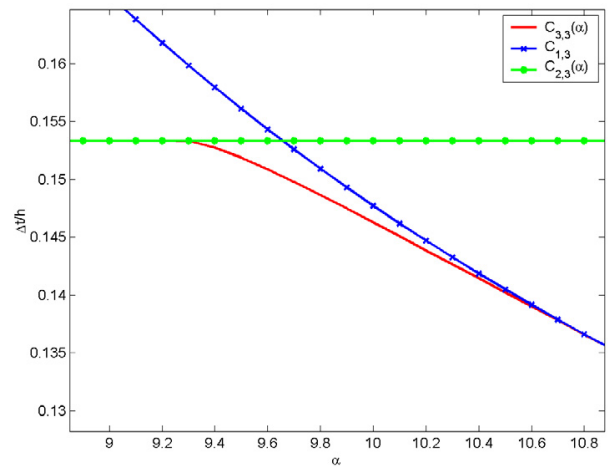

Figure 7 . Zoom on the 3 cond. for $p=3$.

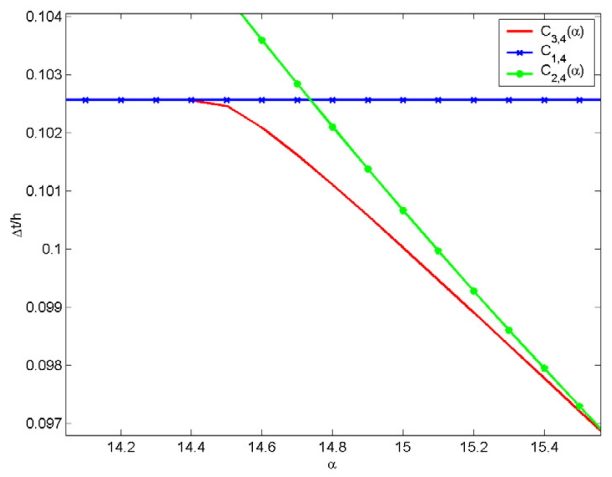

Figure 9. Zoom on the 3 cond. for $p=4$.

CFL condition in a small segment around $\alpha_{1, p}$. The behaviour is confirmed in Figures 3, 5, 7, 9 and 11 which represent a zoom around $\alpha_{1, p}$. Theses numerical results confirm the fact that Theorem 3.2 provides actually a necessary and sufficient condition except for a small range of $\alpha$. Moreover, the CFL condition remains constant for $\alpha$ from $\frac{p(p+1)}{2}$ to a close value of $\alpha_{p}^{1}$, which means that it is not necessary to choose $\alpha=\frac{p(p+1)}{2}$ to optimize the time step.

\subsection{Comparison with numerical experiments}

In this section, we compare the results we have obtained previously with numerical experiments. The goal are (a) to show that the necessary condition we have proposed is actually sufficient in practical applications and (b) to show that even if the results we have proposed have been obtained for infinite domains, they are also valid for finite domains. We consider the simulation of wave propagation in an homogeneous $1 \mathrm{D}$ domain $\Omega=[0,10]$ with a velocity $c=(\mu / \rho)^{1 / 2}=1 \mathrm{~ms}^{-1}$. We impose also Dirichlet boundary conditions at the both ends of the domain and the length of the space step is $h=0.1$. We precise that we have performed these experiments with other boundary conditions as Neumann or periodic boundary coonditions and the results are equivalent. 


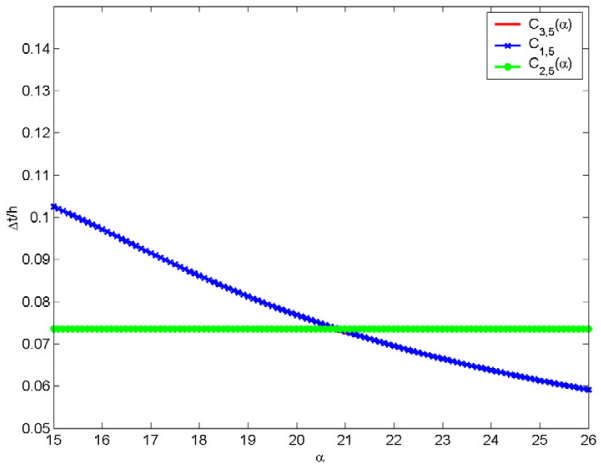

Figure 10. The 3 conditions for $p=5$.

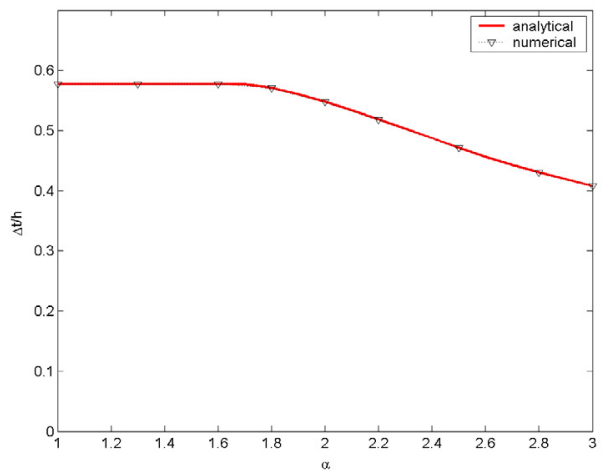

FiguRE 12. Numerical comparison in $P^{1}$.

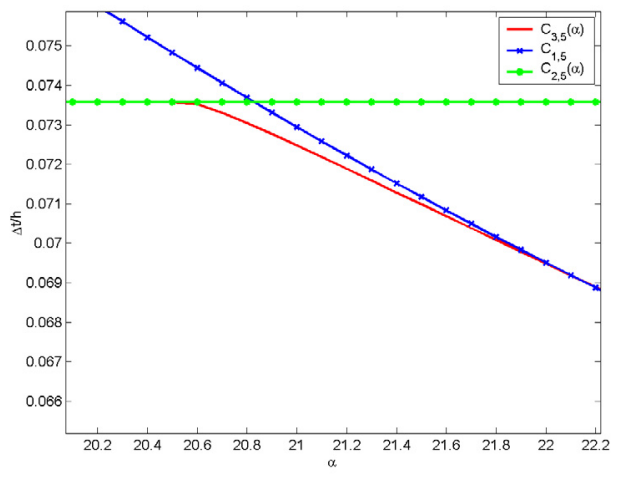

Figure 11. Zoom on the 3 cond. for $p=5$.

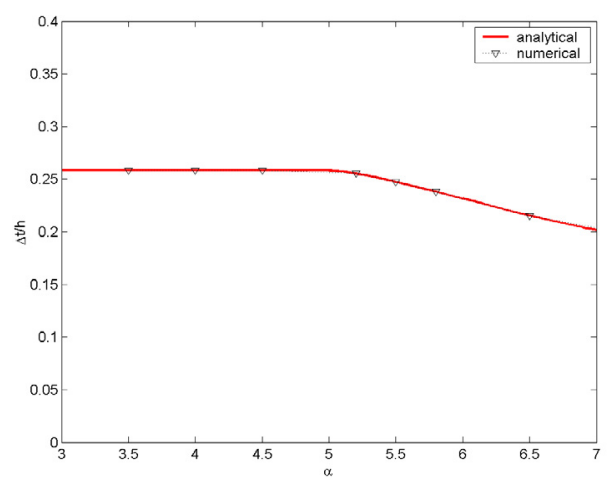

FiguRE 13. Numerical comparison in $P^{2}$.

We computed numerically the greatest eigenvalue $\lambda_{\max }$ of the matrix $M^{-1} K$ and we deduced the CFL condition of the scheme using the formula $\frac{c \Delta t}{h} \leq \frac{2}{\sqrt{\lambda_{\max }}}$. In Figures 12, 13, 14, 15 and 16 we compare the analytical CFL (red line) obtained by Theorem 3.4 to the numerical CFL (triangles), respectively for $p=1,2,3,4$ and 5. All figures show a very good agreement between the analytical and the numerical CFL.

\section{CONCLUSiON}

In this paper, we have proved necessary conditions for $L^{2}$-stability of an IPDG method using regular meshes and the numerical results show that these conditions are actually sufficient in practice. It also confirm the conjecture of Ainsworth, Monk and Muniz up to $p=5$. Moreover, we have observed that the CFL condition is constant with respect to $\alpha$ on a segment $\left[\frac{p(p+1)}{2}, \tilde{\alpha}\right]$ and is decreasing as $\alpha^{-1 / 2}$ for $\alpha>\tilde{\alpha}$. This means that it is not necessary to choose $\alpha$ too close to $p(p+1) / 2$ to improve the CFL condition. Finally, we have observed that a good choice for $\xi_{F}$ should be the diameter of the inscribed circle (or sphere). This should be confirmed by an analysis on triangular meshes, which will be the topic of future work. 


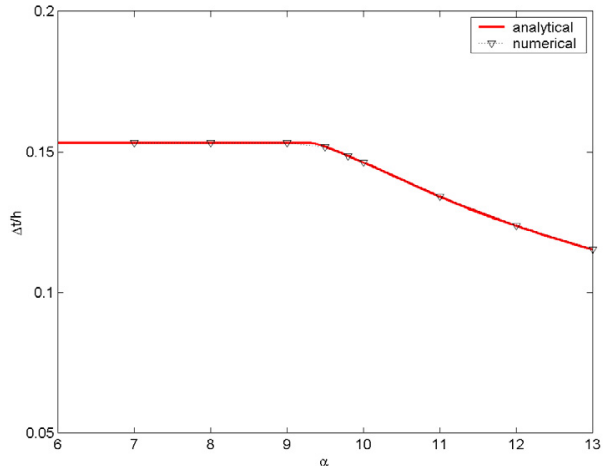

FiguRE 14. Numerical comparison in $P^{3}$.

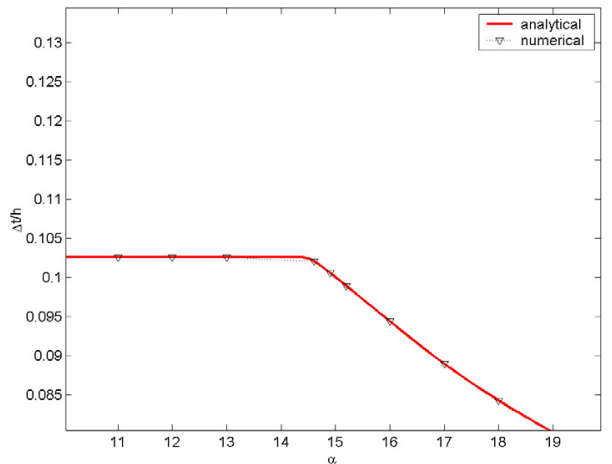

FiguRE 15. Numerical comparison in $P^{4}$.

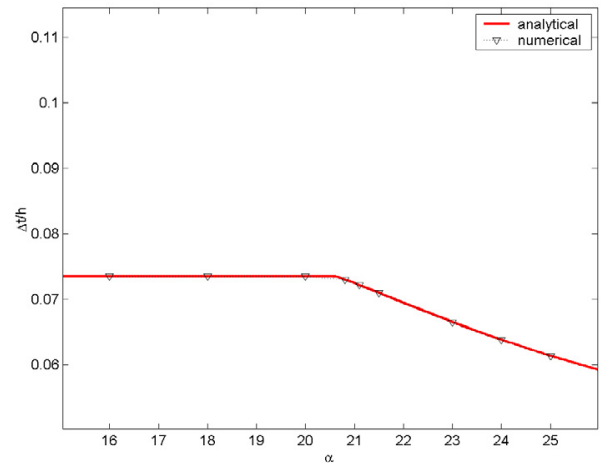

Figure 16. Numerical comparison in $P^{5}$.

\section{Appendix A. Expression of the polynomial $q_{\alpha}$}

- In the case of discontinuous basis functions of degree 1 , we can easily obtain the following characteristic polyomial associated to the matrix $N_{\beta}$

$$
q_{\alpha}(\beta, \lambda)=\lambda^{2}+c_{1}(\alpha, \beta) \lambda+c_{0}(\alpha, \beta)
$$

with

$$
\left\{\begin{array}{l}
c_{1}(\alpha, \beta)=\frac{4}{h^{2}}((3-\alpha) \cos (\beta)-2 \alpha) \\
c_{0}(\alpha, \beta)=\frac{12}{h^{4}}\left(\cos ^{2}(\beta)-2 \alpha \cos (\beta)+2 \alpha-1\right) .
\end{array}\right.
$$

- In the case of discontinuous basis functions of degree 2 , the characteristic polyomial associated to the matrix $N_{\beta}$ is

$$
q_{\alpha}(\beta, \lambda)=-\lambda^{3}+c_{2}(\alpha, \beta) \lambda^{2}+c_{1}(\alpha, \beta) \lambda+c_{0}(\alpha, \beta)
$$


with

$$
\left\{\begin{array}{l}
c_{2}(\alpha, \beta)=-\frac{6}{h^{2}}((\alpha-8) \cos (\beta)-3 \alpha) \\
c_{1}(\alpha, \beta)=\frac{12}{h^{4}}\left(-6 \cos ^{2}(\beta)-2(15+4 \alpha) \cos (\beta)+4(24-13 \alpha)\right) \\
c_{0}(\alpha, \beta)=-\frac{1440}{h^{6}}\left(\cos ^{2}(\beta)+(\alpha-3) \cos (\beta)+2-\alpha\right) .
\end{array}\right.
$$

- In the case of discontinuous basis functions of degree 4 , the characteristic polyomial associated to the matrix $N_{\beta}$ is

$$
q_{\alpha}(\beta, \lambda)=-\lambda^{5}+c_{4}(\alpha, \beta) \lambda^{4}+c_{3}(\alpha, \beta) \lambda^{3}+c_{2}(\alpha, \beta) \lambda^{2}+c_{1}(\alpha, \beta) \lambda+c_{0}(\alpha, \beta)
$$

with

$$
\left\{\begin{array}{l}
c_{4}(\alpha, \beta)=-\frac{10}{h^{2}}((\alpha-24) \cos (\beta)-5 \alpha) \\
c_{3}(\alpha, \beta)=-\frac{120}{h^{4}}\left(5 \cos ^{2}(\beta)+(4 \alpha+287) \cos (\beta)+10(15 \alpha-88) \alpha\right) \\
c_{2}(\alpha, \beta)=-\frac{10080}{h^{6}}\left(5 \cos ^{2}(\beta)+(3 \alpha-305) \cos (\beta)+990-133 \alpha\right) \\
c_{1}(\alpha, \beta)=-\frac{201600}{h^{8}}\left(15 \cos ^{2}(\beta)+(8 \alpha+165) \cos (\beta)+2(59 \alpha-468)\right) \\
c_{0}(\alpha, \beta)=-\frac{50803200}{h^{10}}\left(2 \cos ^{2}(\beta)+(\alpha-10) \cos (\beta)+8-\alpha\right) .
\end{array}\right.
$$

- In the case of discontinuous basis functions of degree 5 , the characteristic polyomial associated to the matrix $N_{\beta}$ is

$$
q_{\alpha}(\beta, \lambda)=\lambda^{6}+c_{5}(\alpha, \beta) \lambda^{5}+c_{4}(\alpha, \beta) \lambda^{4}+c_{3}(\alpha, \beta) \lambda^{3}+c_{2}(\alpha, \beta) \lambda^{2}+c_{1}(\alpha, \beta) \lambda+c_{0}(\alpha, \beta)
$$

with

$$
\left\{\begin{array}{l}
c_{5}(\alpha, \beta)=-\frac{12}{h^{2}}((\alpha-35) \cos (\beta)+6 \alpha) \\
c_{4}(\alpha, \beta)=-\frac{420}{h^{4}}\left(-3 \cos ^{2}(\beta)+(2 \alpha+336) \cos (\beta)+1155-134 \alpha\right) \\
c_{3}(\alpha, \beta)=-\frac{40320}{h^{6}}\left(-4 \cos ^{2}(\beta)+(2 \alpha-702) \cos (\beta)+256 \alpha-2849 \alpha\right) \\
c_{2}(\alpha, \beta)=-\frac{1814400}{h^{8}}\left(-9 \cos ^{2}(\beta)+(4 \alpha 770) \cos (\beta)+9343-326 \alpha\right) \\
c_{1}(\alpha, \beta)=-\frac{101606400}{h^{10}}\left(-12 \cos ^{2}(\beta)+(5 \alpha-303) \cos (\beta)+94 \alpha-1170\right) \\
c_{0}(\alpha, \beta)=-\frac{10059033600}{h^{12}}\left(-5 \cos ^{2}(\beta)+(2 \alpha-20) \cos (\beta)+25-2 \alpha\right)
\end{array}\right.
$$

\section{Appendix B. Definition of $Q_{p, \alpha}$ AND $\tilde{Q}_{p, \alpha}$}

We present here the expressions of the polynomial $Q_{p, \alpha}$ and the rational function $\tilde{Q}_{p, \alpha}$ for $1 \leq p \leq 5$.

- For the polynomials of degree $1, \tilde{Q}_{1, \alpha}$ is defined by

$$
\tilde{Q}_{1, \alpha}(\lambda)=\frac{h^{2} \lambda}{2}\left(\frac{\alpha}{3}-1\right)+\alpha .
$$


We have,

$$
Q_{1, \alpha}(\lambda)=\sum_{i=0}^{2} \lambda^{i} h^{2 i} \tilde{c}_{i}(\alpha)
$$

with

$$
\left\{\begin{array}{l}
\tilde{c}_{0}(\alpha)=36\left(\alpha^{2}-2 \alpha+1\right), \\
\tilde{c}_{1}(\alpha)=12\left(\alpha^{2}-\alpha\right), \\
\tilde{c}_{2}(\alpha)=\alpha^{2}-6 \alpha+6
\end{array}\right.
$$

- In the case $p=2$, the definition of $\tilde{Q}_{p, \alpha}$ is

$$
\tilde{Q}_{2, \alpha}(\lambda)=-\frac{(\alpha-1) h^{4} \lambda^{2}+4(15+4 \alpha) h^{2} \lambda+240(\alpha-3)}{24\left(h^{2} \lambda+20\right)} .
$$

The polynomial $Q_{2, \alpha}$ is such that

$$
Q_{2, \alpha}(\lambda)=\sum_{i=0}^{4} \lambda^{i} h^{2 i} \tilde{c}_{i}(\alpha)
$$

with

$$
\left\{\begin{array}{l}
\tilde{c}_{0}(\alpha)=57600\left(\alpha^{2}-2 \alpha+1\right) \\
\tilde{c}_{1}(\alpha)=1920\left(4 \alpha^{2}-43 \alpha+39\right) \\
\tilde{c}_{2}(\alpha)=16\left(46 \alpha^{2}-342 \alpha+1521\right) \\
\tilde{c}_{3}(\alpha)=8\left(4 \alpha^{2}+\alpha-140\right) \\
\tilde{c}_{4}(\alpha)=\alpha^{2}-16 \alpha+56
\end{array}\right.
$$

- For $p=3$, we have

$$
Q_{3, \alpha}(\lambda)=\sum_{i=0}^{6} \lambda^{i} h^{2 i} \tilde{c}_{i}(\alpha)
$$

with

$$
\left\{\begin{array}{l}
\tilde{c}_{0}(\alpha)=635040000\left(\alpha^{2}-12 \alpha+36\right) \\
\tilde{c}_{1}(\alpha)=3628800\left(15 \alpha^{2}+70 \alpha-96\right) \\
\tilde{c}_{2}(\alpha)=86400\left(31 \alpha^{2}-447 \alpha+5316\right) \\
\tilde{c}_{3}(\alpha)=14400\left(8 \alpha^{2}-135 \alpha-1728\right) \\
\tilde{c}_{4}(\alpha)=180\left(17 \alpha^{2}-442 \alpha+7740\right) \\
\tilde{c}_{5}(\alpha)=60\left(\alpha^{2}+16 \alpha-357\right) \\
\tilde{c}_{6}(\alpha)=\alpha^{2}-30 \alpha+210
\end{array}\right.
$$

and $\tilde{Q}_{3, \alpha}$ is defined by

$$
\tilde{Q}_{3, \alpha}(\lambda)=\frac{(\alpha-15) h^{6} \lambda^{3}+30(23+\alpha) h^{4} \lambda^{2}+360(3 \alpha-65) h^{2} \lambda+25200(\alpha-3)}{60\left(h^{4} \lambda^{2}+48 h^{2} \lambda+1260\right)} .
$$


- For the polynomials of degree $4, \tilde{Q}_{4, \alpha}$ is defined by

$$
\tilde{Q}_{4, \alpha}(\lambda)=-\frac{\tilde{B}_{4, \alpha}(\lambda)}{120\left(169344+h^{6} \lambda^{3}+84 h^{4} \lambda^{2}+5040 h^{2} \lambda\right)}
$$

with

$$
\begin{aligned}
\tilde{B}_{4, \alpha}(\lambda)= & \lambda^{4} h^{8}(\alpha-24)+12 \lambda^{3} h^{6}(4 \alpha+287)+1008 \lambda^{2} h^{4}(3 \alpha-305) \\
& +20160 \lambda h^{2}(8 \alpha-165)+5080320(\alpha-1)
\end{aligned}
$$

and, we have

$$
Q_{4, \alpha}(\lambda)=\sum_{i=0}^{8} \lambda^{i} h^{2 i} \tilde{c}_{i}(\alpha) .
$$

with

$$
\left\{\begin{array}{l}
\tilde{c}_{0}(\alpha)=25809651302400(\alpha-6)^{2}, \\
\tilde{c}_{1}(\alpha)=204838502400\left(8 \alpha^{2}-357 \alpha+1854\right), \\
\tilde{c}_{2}(\alpha)=81285120\left(698 \alpha^{2}+3882 \alpha+292185\right), \\
\tilde{c}_{3}(\alpha)=203212800\left(72 \alpha^{2}-13791 \alpha-328\right), \\
\tilde{c}_{4}(\alpha)=48384\left(719 \alpha^{2}-12750 \alpha+2419275\right), \\
\tilde{c}_{5}(\alpha)=8064\left(76 \alpha^{2}-972 \alpha-286209\right), \\
\tilde{c}_{6}(\alpha)=144\left(58 \alpha^{2}-5282 \alpha+201609\right), \\
\tilde{c}_{7}(\alpha)=24\left(4 \alpha^{2}+241 \alpha-6972\right), \\
\tilde{c}_{8}(\alpha)=\alpha^{2}-48 \alpha+55 .
\end{array}\right.
$$

- For $p=5, \tilde{Q}_{5, \alpha}$ is such that

$$
\tilde{Q}_{5, \alpha}(\lambda)=\frac{\tilde{B}_{5, \alpha}(\lambda)}{210\left(39916800+\lambda^{4} h^{8}+128 \lambda^{3} h^{6}+12960 \lambda^{2} h^{4}+967680 \lambda h^{2}\right)}
$$

with

$$
\begin{aligned}
\tilde{B}_{5, \alpha}(\lambda)= & \lambda^{5} h^{10}(\alpha-35)+70 \lambda^{4} h^{8}(\alpha+168)+6720 \lambda^{3} h^{6}(\alpha-351) \\
& +302400 \lambda^{2} h^{4}(2 \alpha+385)+8467200 \lambda h^{2}(5 \alpha-303)+1676505600(\alpha-1)
\end{aligned}
$$

and we have

$$
Q_{5, \alpha}(\lambda)=\sum_{i=0}^{10} \lambda^{i} h^{2 i} \tilde{c}_{i}(\alpha)
$$


with

$$
\left\{\begin{array}{l}
\tilde{c}_{0}(\alpha)=2810671026831360000(\alpha-15)^{2}, \\
\tilde{c}_{1}(\alpha)=28390616432640000(5 \alpha+168)(\alpha-15), \\
\tilde{c}_{2}(\alpha)=10241925120000\left(373 \alpha^{2}-35067 \alpha+855423\right), \\
\tilde{c}_{3}(\alpha)=3072577536000\left(24 \alpha^{2}-895 \alpha-159240\right), \\
\tilde{c}_{4}(\alpha)=1016064000\left(1151 \alpha^{2}-11360 \alpha+24379995\right), \\
\tilde{c}_{5}(\alpha)=67737600\left(257 \alpha^{2}-3570 \alpha-9096540\right), \\
\tilde{c}_{6}(\alpha)=2822400\left(76 \alpha^{2}-2417 \alpha+2988895\right), \\
\tilde{c}_{7}(\alpha)=67200\left(32 \alpha^{2}+2383 \alpha-974820\right), \\
\tilde{c}_{8}(\alpha)=140\left(131 \alpha^{2}-37062 \alpha+2285235\right), \\
\tilde{c}_{9}(\alpha)=140\left(\alpha^{2}+151 \alpha-5912\right), \\
\tilde{c}_{10}(\alpha)=\alpha^{2}-70 \alpha+1190 .
\end{array}\right.
$$

\section{Appendix C. Proof of Theorem 5.1}

This section is devoted to the proof of the following theorem

Theorem C.1. For all $\mathbf{m}=\left(m_{k}\right)_{k=1, \ldots, 3} \in\{1, \ldots, p+1\}^{3}$ and $\mathbf{n}=\left(n_{k}\right)_{k=1, \ldots, 3} \in\{1, \ldots, p+1\}^{3}$, we have

$$
\begin{aligned}
& \text { 1. } M_{3, p}(\mathbf{m}, \mathbf{n})=\prod_{i=1}^{3} M_{1, p}\left(m_{i}, n_{i}\right), \\
& \text { 2. } K_{3, p}(\mathbf{m}, \mathbf{n})=\sum_{i=1}^{3}\left(K_{1, p}\left(m_{i}, n_{i}\right) \prod_{k=1, k \neq i}^{3} M_{1, p}\left(m_{k}, n_{k}\right)\right) \\
& \text { 3. } K_{3, p}^{C}(\mathbf{m}, \mathbf{n})=K_{1, p}^{W}\left(m_{p_{C}}, n_{p_{C}}\right) \prod_{k=1, k \neq p_{C}}^{3} M_{1, p}\left(m_{k}, n_{k}\right) \\
& \text { 4. } N_{3, p}(\mathbf{m}, \mathbf{n})=\sum_{p=1}^{3} N_{1, p}\left(m_{p}, n_{p}\right) \prod_{k=1, k \neq p}^{3} \delta_{m_{k}, n_{k}} \\
& \text { 5. } N_{3, p}^{C}(\mathbf{m}, \mathbf{n})=N_{1, p}^{W}\left(m_{p_{C}}, n_{p_{C}}\right) \prod_{k=1, k \neq p_{C}}^{3} \delta_{m_{k}, n_{k}}
\end{aligned}
$$

$$
\text { where } p_{C}=\left\{\begin{array}{l}
1 \text { if } C \in\{E, W\} ; \\
2 \text { if } C \in\{N, S\}, \quad \text { and } N_{1, p}=M_{1, p}^{-1} K_{1, p} \text { and } N_{1, p}^{C}=M_{1, p}^{-1} K_{1, p}^{C} ; \\
3 \text { if } C \in\{B, F\} .
\end{array}\right.
$$


Proof.

- Proof of 1.

Considering the notations and the results of Section 5, we have

$$
\begin{aligned}
M_{3, p}(\mathbf{m}, \mathbf{n}) & =h^{3} \int_{\hat{K}} \hat{\varphi}_{\mathbf{m}} \hat{\varphi}_{\mathbf{n}} \mathrm{d} \mathbf{x} \\
& =h^{3} \int_{\hat{K}} \prod_{i=1}^{3} \hat{\varphi}_{m_{i}}\left(x_{i}\right) \prod_{i=1}^{3} \hat{\varphi}_{n_{i}}\left(x_{i}\right) \prod_{i=1}^{3} \mathrm{~d} x_{i} \\
& =\prod_{i=1}^{3} h \int_{[0,1]} \hat{\varphi}_{m_{i}}\left(x_{i}\right) \hat{\varphi}_{n_{i}}\left(x_{i}\right) \mathrm{d} x_{i} \\
& =\prod_{i=1}^{3} M_{1, p}\left(m_{i}, n_{i}\right)
\end{aligned}
$$

- Proof of 2.

We first have the following lemma for the volumic term.

Lemma C.2. For all $\mathbf{m}=\left(m_{k}\right)_{k=1, \ldots, 3} \in\{1, \ldots, p+1\}^{3}$ and $\mathbf{n}=\left(n_{k}\right)_{k=1, \ldots, 3} \in\{1, \ldots, p+1\}^{3}$, we have

$$
h \int_{\hat{K}} \nabla \hat{\varphi}_{\mathbf{m}} \cdot \nabla \hat{\varphi}_{\mathbf{n}} \mathrm{d} \mathbf{x}=\sum_{i=1}^{3}\left(\frac{1}{h} \int_{[0,1]} \frac{\partial \hat{\varphi}_{m_{i}}}{\partial x_{i}}\left(x_{i}\right) \frac{\partial \hat{\varphi}_{n_{i}}}{\partial x_{i}}\left(x_{i}\right) \mathrm{d} x_{i} \prod_{k=1, k \neq i}^{3} M_{1, p}\left(m_{k}, n_{k}\right)\right) .
$$

Proof. We know that, $\forall \mathbf{m} \in\{1, \ldots, p+1\}^{d}$

$$
\hat{\varphi}_{\mathbf{m}}(\mathbf{x})=\prod_{k=1}^{3} \hat{\varphi}_{m_{p}}\left(x_{p}\right)
$$

which implies that

$$
\frac{\partial \hat{\varphi}_{\mathbf{m}}}{\partial x_{k}}(\mathbf{x})=\frac{\partial \hat{\varphi}_{m_{k}}}{\partial x_{k}}\left(x_{k}\right) \prod_{i=1, i \neq k}^{3} \hat{\varphi}_{m_{i}}\left(x_{i}\right) .
$$

Then, using the same reasoning as previously,

$$
\begin{aligned}
h \int_{\hat{K}} \nabla \hat{\varphi}_{\mathbf{m}} \cdot \nabla \hat{\varphi}_{\mathbf{n}} \mathrm{d} \mathbf{x} & =\sum_{i=1}^{3}\left(\frac{1}{h} \int_{[0,1]} \frac{\partial \hat{\varphi}_{m_{i}}}{\partial x_{i}}\left(x_{i}\right) \frac{\partial \hat{\varphi}_{n_{i}}}{\partial x_{i}}\left(x_{i}\right) \mathrm{d} x_{i} \prod_{k=1, k \neq i}^{3} h \int_{[0,1]} \hat{\varphi}_{m_{k}}\left(x_{k}\right) \hat{\varphi}_{n_{k}}\left(x_{k}\right) \mathrm{d} x_{k}\right) \\
& =\sum_{i=1}^{3}\left(\frac{1}{h} \int_{[0,1]} \frac{\partial \hat{\varphi}_{m_{i}}}{\partial x_{i}}\left(x_{i}\right) \frac{\partial \hat{\varphi}_{n_{i}}}{\partial x_{i}}\left(x_{i}\right) \mathrm{d} x_{i} \prod_{k=1, k \neq i}^{3} M_{1, p}\left(m_{k}, n_{k}\right)\right)
\end{aligned}
$$

which ends the proof.

Now, we have to deal with the surface terms.

Let us first remark that, over all the faces $\Gamma^{C}$,

$$
\left.\nabla \hat{\varphi}_{\mathbf{m}} \cdot \nu\right|_{\Gamma^{C}}=\frac{\partial \hat{\varphi}_{m_{p_{C}}}}{\partial x_{p_{C}}}\left(x_{p_{C}}\right) \nu_{1, C} \prod_{k=1, k \neq p_{C}}^{3} \hat{\varphi}_{m_{k}}\left(x_{k}\right)
$$


where $\nu_{1, C}$ is the outward unit normal vector in the one dimensional case defined by

$$
\nu_{1, C}=\left\{\begin{array}{rll}
1 & \text { if } & C \in\{E, N, F\} \\
-1 & \text { if } \quad C \in\{W, S, B\}
\end{array}\right.
$$

and $x_{p_{C}}$ is defined by

$$
x_{p_{C}}=\left\{\begin{array}{lll}
1 & \text { if } & C \in\{E, N, F\} \\
0 & \text { if } & C \in\{W, S, B\} .
\end{array}\right.
$$

Then, we can propose the following lemma.

Lemma C.3. For all $C \in\{E, W, N, S, F, B\}$, we have $\forall \mathbf{m}, \mathbf{n} \in\{1, \ldots, p+1\}^{d}$

$$
\begin{aligned}
h \int_{\Gamma^{C}} \hat{\varphi}_{\mathbf{m}}\left(\nabla \hat{\varphi}_{\mathbf{n}} \cdot \nu\right) \mathrm{d} \sigma & =\frac{1}{h} \hat{\varphi}_{m_{p_{C}}}\left(x_{p_{C}}\right) \frac{\partial \hat{\varphi}_{n_{p_{C}}}}{\partial x_{p_{C}}}\left(x_{p_{C}}\right) \nu_{1, C} \prod_{k=1, k \neq p_{C}}^{3} M_{1, p}\left(m_{k}, n_{k}\right) \\
h^{2} \int_{\Gamma^{C}} \gamma \hat{\varphi}_{\mathbf{m}} \hat{\varphi}_{\mathbf{n}} \mathrm{d} \sigma & =\gamma \hat{\varphi}_{m_{p_{C}}}\left(x_{p_{C}}\right) \hat{\varphi}_{n_{p_{C}}}\left(x_{p_{C}}\right) \prod_{k=1, k \neq p_{C}}^{3} M_{1, p}\left(m_{k}, n_{k}\right) .
\end{aligned}
$$

Proof. First of all, using (C.2), we have

$$
\begin{aligned}
h \int_{\Gamma^{C}} \hat{\varphi}_{\mathbf{m}}\left(\nabla \hat{\varphi}_{\mathbf{n}} \cdot \nu\right) \mathrm{d} \sigma & =h \int_{\Gamma^{C}}\left(\prod_{k=1}^{3} \hat{\varphi}_{m_{k}}\left(x_{k}\right)\right)\left(\frac{\partial \hat{\varphi}_{n_{p_{C}}}}{\partial x_{p_{C}}}\left(x_{p_{C}}\right) \nu_{1, C} \prod_{k=1, k \neq p_{C}}^{3} \hat{\varphi}_{n_{k}}\left(x_{k}\right)\right) \prod_{k=1, k \neq p_{C}}^{3} \mathrm{~d} x_{k} \\
& =\frac{1}{h} \hat{\varphi}_{m_{p_{C}}}\left(x_{p_{C}}\right) \frac{\partial \hat{\varphi}_{n_{p_{C}}}}{\partial x_{p_{C}}}\left(x_{p_{C}}\right) \nu_{1, C} \prod_{k=1, k \neq p_{C}}^{3} h^{2} \int_{[0,1]} \hat{\varphi}_{m_{k}}\left(x_{k}\right) \hat{\varphi}_{n_{k}}\left(x_{k}\right) \mathrm{d} x_{k}
\end{aligned}
$$

which can be rewritten as

$$
\int_{\Gamma^{C}} \hat{\varphi}_{\mathbf{m}}\left(\nabla \hat{\varphi}_{\mathbf{n}} \cdot \nu\right) \mathrm{d} \sigma=\hat{\varphi}_{m_{p_{C}}}\left(x_{p_{C}}\right) \frac{\partial \hat{\varphi}_{n_{p_{C}}}}{\partial x_{p_{C}}}\left(x_{p_{C}}\right) \nu_{1, C} \prod_{k=1, k \neq p_{C}}^{3} M_{1, p}\left(m_{k}, n_{k}\right) .
$$

In the same way, for the penalization term, we have:

$$
\begin{aligned}
h^{2} \int_{\Gamma^{C}} \gamma \hat{\varphi}_{\mathbf{m}} \hat{\varphi}_{\mathbf{n}} \mathrm{d} \sigma & =\hat{\varphi}_{m_{p_{C}}}\left(x_{p_{C}}\right) \hat{\varphi}_{n_{p_{C}}}\left(x_{p_{C}}\right) h^{2} \int_{\Gamma^{C}} \gamma \prod_{k=1, k \neq p_{C}}^{3} \hat{\varphi}_{m_{k}}\left(x_{k}\right) \prod_{k=1, k \neq p_{C}}^{3} \hat{\varphi}_{n_{k}}\left(x_{k}\right) \prod_{k=1, k \neq p_{C}}^{3} \mathrm{~d} \sigma_{k} \\
& =\gamma \hat{\varphi}_{m_{p_{C}}}\left(x_{p_{C}}\right) \hat{\varphi}_{n_{p_{C}}}\left(x_{p_{C}}\right) \prod_{k=1, k \neq p_{C}}^{3} h^{2} \int_{[0,1]} \hat{\varphi}_{m_{k}}\left(x_{k}\right) \hat{\varphi}_{n_{k}}\left(x_{k}\right) \mathrm{d} x_{k}
\end{aligned}
$$

which clearly implies that

$$
\int_{\Gamma^{C}} \gamma \hat{\varphi}_{\mathbf{m}} \hat{\varphi}_{\mathbf{n}} \mathrm{d} \sigma=\gamma \hat{\varphi}_{m_{p_{C}}}\left(x_{p_{C}}\right) \hat{\varphi}_{n_{p_{C}}}\left(x_{p_{C}}\right) \prod_{k=1, k \neq p_{C}}^{3} M_{1, p}\left(m_{k}, n_{k}\right)
$$

which ends the proof.

Finally, using the two lemmas, we obtain

$$
K_{3, p}(\mathbf{m}, \mathbf{n})=\sum_{i=1}^{3}\left(K_{1, p}\left(m_{i}, n_{i}\right) \prod_{k=1, k \neq i}^{3} M_{1, p}\left(m_{k}, n_{k}\right)\right) .
$$


- Proof of 3.

To rewrite the terms $K_{3, p}^{C}(\mathbf{m}, \mathbf{n})$ for all $\mathbf{m}, \mathbf{n}$, we use a similar reasoning as for $K_{3, p}$.

- Proof of 4 .

To prove 4. and 5., we need the following lemma.

Lemma C.4. Let $\mathbf{m}=\left(m_{p}\right)_{p=1, \ldots, 3}$ and $\mathbf{n}=\left(n_{p}\right)_{p=1, \ldots, 3}$.

We have

$$
M_{3, p}^{-1}(\mathbf{m}, \mathbf{n})=\prod_{k=1}^{3} M_{1, p}^{-1}\left(m_{k}, n_{k}\right)
$$

Proof. Let $A$ be the matrix defined $\forall \mathbf{m}, \mathbf{n} \in\{1, \ldots, p+1\}^{d}$ by

$$
A(\mathbf{m}, \mathbf{n})=\prod_{k=1}^{3} M_{1, p}^{-1}\left(m_{k}, n_{k}\right)
$$

We have

$$
\begin{aligned}
(A M)(\mathbf{m}, \mathbf{n}) & =\sum_{l_{1}, \ldots, l_{3}=1}^{p+1}\left(\prod_{k=1}^{3} M_{1, p}^{-1}\left(m_{k}, l_{k}\right) \prod_{k=1}^{3} M_{1, p}\left(l_{k}, n_{k}\right)\right) \\
& =\left(\sum_{l_{1}, l_{2}=1}^{p+1}\left(\prod_{k=1}^{2} M_{1, p}^{-1}\left(m_{k}, l_{k}\right) \prod_{k=1}^{2} M_{1, p}\left(l_{k}, n_{k}\right)\right) \sum_{l_{3}=1}^{3} M_{1, p}^{-1}\left(m_{3}, l_{3}\right) M_{1, p}\left(l_{3}, n_{3}\right)\right) \\
& =\prod_{k=1}^{3}\left(\sum_{l_{k}=1}^{p+1} M_{1, p}^{-1}\left(m_{k}, l_{k}\right) M_{1, p}\left(l_{k}, n_{k}\right)\right) .
\end{aligned}
$$

But,

$$
\sum_{l_{k}=1}^{p+1} M_{1, p}^{-1}\left(m_{k}, l_{k}\right) M_{1, p}\left(l_{k}, n_{k}\right)=\left(M_{1, p}^{-1} M_{1, p}\right)\left(m_{k}, n_{k}\right)=\delta_{m_{k}, n_{k}}
$$

so that

$$
(A M)(\mathbf{m}, \mathbf{n})=\prod_{k=1}^{3} \delta_{m_{k}, n_{k}}=I(\mathbf{m}, \mathbf{n})
$$

where $I$ is the identity matrix, which ends the proof.

Let us now consider the matrix $N_{3, p}=M_{3, p}^{-1} K_{3, p}$. First, we rewrite $K_{3, p}$ as

$$
K_{3, p}=\sum_{q=1}^{3} T_{q}
$$

with $T_{q}(\mathbf{m}, \mathbf{n})=K_{1, p}\left(m_{q}, n_{q}\right) \prod_{k=1, k \neq q}^{3} M_{1, p}\left(m_{k}, n_{k}\right)$. 
Then, $M_{3, p}^{-1} K_{3, p}=\sum_{q=1}^{3} M_{3, p}^{-1} T_{q}$ and, using Lemma C.4

$$
\begin{aligned}
\left(M_{3, p}^{-1} T_{1}\right)(\mathbf{m}, \mathbf{n}) & =\sum_{l_{1}, \ldots, l_{3}=1}^{p+1}\left(\prod_{k=1}^{3} M_{1, p}^{-1}\left(m_{k}, l_{k}\right) K_{1, p}\left(l_{1}, n_{1}\right) \prod_{k=2}^{3} M_{1, p}\left(l_{k}, n_{k}\right)\right) \\
& =\sum_{l_{1}, \ldots, l_{3}=1}^{p+1} M_{1, p}^{-1}\left(m_{1}, l_{1}\right) K_{1, p}\left(l_{1}, n_{1}\right) M_{1, p}^{-1}\left(m_{2}, l_{2}\right) M_{1, p}\left(l_{2}, n_{2}\right) M_{1, p}^{-1}\left(m_{3}, l_{3}\right) M_{1, p}\left(l_{3}, n_{3}\right) \\
& =\sum_{l_{1}=1}^{p+1} M_{1, p}^{-1}\left(m_{1}, l_{1}\right) K_{1, p}\left(l_{1}, n_{1}\right) \prod_{k=2}^{3}\left(\sum_{l_{k}=1}^{p+1} M_{1, p}^{-1}\left(m_{k}, l_{k}\right) M_{1, p}\left(l_{k}, n_{k}\right)\right) .
\end{aligned}
$$

So that

$$
\left(M_{3, p}^{-1} T_{1}\right)(\mathbf{m}, \mathbf{n})=N_{1, p}\left(m_{1}, n_{1}\right) \delta_{m_{2}, n_{2}} \delta_{m_{3}, n_{3}}
$$

Performing the same calculations for $T_{2}$ and $T_{3}$, we obtain

$$
N_{3, p}(\mathbf{m}, \mathbf{n})=\sum_{p=1}^{3} N_{1, p}\left(m_{p}, n_{p}\right) \prod_{k=1, k \neq p}^{3} \delta_{m_{k}, n_{k}} .
$$

- Proof of 5 .

We apply the technique used to prove 4 . to show that

$$
N_{3, p}^{C}(\mathbf{m}, \mathbf{n})=N_{1, p}^{W}\left(m_{p_{C}}, n_{p_{C}}\right) \prod_{k=1, k \neq p_{C}}^{3} \delta_{m_{k}, n_{k}}
$$

Acknowledgements. We want to thank Hélène Barucq for her helpful advices which allow us to clarify various parts of this article.

\section{REFERENCES}

[1] C. Agut and J. Diaz, Stability analysis of the interior penalty discontinuous Galerkin method for the wave equation. INRIA Res. Report (2010).

[2] M. Ainsworth, P. Monk and W. Muniz, Dispersive and dissipative properties of discontinuous Galerkin finite element methods for the second-order wave equation. J. Sci. Comput. 27 (2006).

[3] D.N. Arnold, An interior penalty finite element method with discontinuous elements. SIAM J. Numer. Anal. 19 (1982) $742-760$.

[4] D.N. Arnold, F. Brezzi, B. Cockburn and L.D. Marini, Unified analysis of disconitnuous Galerkin methods for elliptic problems. SIAM J. Numer. Anal. 39 (2002) 1749-1779.

[5] C. Baldassari, Modélisation et simulation numérique pour la migration terrestre par équation d'ondes. Ph.D. Thesis (2009).

[6] G. Benitez Alvarez, A.F. Dourado Loula, E.G. Dutra do Carmo and A. Alves Rochinha, A discontinuous finite element formulation for Helmholtz equation. Comput. Methods. Appl. Mech. Engrg. 195 (2006) 4018-4035.

[7] G. Cohen, Higher-Order Numerical Methods for Transient Wave Equations. Springer, Berlin (2001).

[8] S. Cohen, P. Joly, J.E. Roberts and N. Tordjman, Higher-order triangular finite elements with mass-lumping for the wave equation. SIAM J. Numer. Anal. 44 (2006) 2408-2431.

[9] S. Cohen, P. Joly and N. Tordjman, Higher-order finite elements with mass-lumping for the 1d wave equation. Finite Elem. Anal. Des. 16 (1994) 329-336.

[10] M.A. Dablain, The application of high order differencing for the scalar wave equation. Geophys. 51 (1986) 54-56.

[11] J.D. De Basabe and M.K. Sen, Stability of the high-order finite elements for acoustic or elastic wave propagation with high-order time stepping. Geophys. J. Int. 181 (2010) 577-590.

[12] Y. Epshteyn and B. Rivière, Estimation of penalty parameters for symmetric interior penalty galerkin methods. J. Comput. Appl. Math. 206 (2007) 843-872.

[13] S. Fauqueux, Eléments finis mixtes spectraux et couches absorbantes parfaitement adaptées pour la propagation d'ondes élastiques en régime transitoire. Ph.D. Thesis (2003). 
[14] J.-C. Gilbert and P. Joly, Higher order time stepping for second order hyperbolic problems and optimal CFL conditions. Comput. Methods Appl. Sci. 16 (2008) 67-93.

[15] M.J. Grote, A. Schneebeli and D. Schötzau, Discontinuous Galerkin finite element method for the wave equation. SIAM J. Numer. Anal. 44 (2006) 2408-2431.

[16] M.J. Grote and D. Schötzau, Convergence analysis of a fully discrete dicontinuous Galerkin method for the wave equation. Preprint No. 2008-04 (2008).

[17] D. Komatitsch and J. Tromp, Introduction to the spectral element method for three-dimensional seismic wave propagation. Geophys J. Int. 139 (1999) 806-822.

[18] P. Lax and B. Wendroff, Systems of conservation laws. Commun. Pure Appl. Math. XIII (1960) $217-237$.

[19] G. Seriani and E. Priolo, Spectral element method for acoustic wave simulation in heterogeneous media. Finite Elem. Anal. Des. 16 (1994) 37-348.

[20] K. Shahbazi, An explicit expression for the penalty parameter of the interior penalty method. J. Comput. Phys. 205 (2005) 401-407.

[21] G.R. Shubin and J.B. Bell, A modified equation approach to constructing fourth-order methods for acoustic wave propagation. SIAM J. Sci. Statist. Comput. 8 (1987) 135-151.

[22] T. Warburton and J.S. Hesthaven, On the constants in $h p$-finite element trace inverse inequalities. Comput. Methods Appl. Mech. Engrg. 192 (2003) 2765-2773. 